\title{
PRESENTATIONS OF GENERALISATIONS OF THOMPSON'S GROUP V
}

\author{
Conchita Martínez-PÉrez, Francesco Matucci And BRita Nucinkis
}

\begin{abstract}
We consider generalisations of Thompson's group $V$, denoted by $V_{r}(\Sigma)$, which also include the groups of Higman, Stein and Brin. We showed earlier (Forum Math. 28:5 (2016), 909-921) that under some mild conditions these groups and centralisers of their finite subgroups are of type $F_{\infty}$. Under more general conditions we show that the groups $V_{r}(\Sigma)$ are finitely generated and, under the mild conditions mentioned above for which they are of type $F_{\infty}$ and hence finitely presented, we give a recipe to find explicit presentations. For the centralisers of finite subgroups we find a suitable infinite presentation and then show how to apply a general procedure to shorten this presentation. In the appendix, we give a proof of this general shortening procedure.
\end{abstract}

\section{Introduction}

The original Thompson groups $F \leq T \leq V$ are groups of homeomorphisms of the unit interval, the circle and the Cantor set respectively. In this note we consider generalisations of these groups, which are described as groups of automorphisms of certain Cantor algebras. These groups include Higman's [1974], Stein's [1992] and Brin's [2004] generalisations of $V$.

The groups $F, T$ and $V$ have attracted the attention of group theorists for several reasons, one of them being that there are nice presentations and ways to represent elements available, making it possible to prove interesting results about metrics, geodesics and decision problems. However, the situation changes when one moves to some of their generalisations. There are presentations available for Higman's groups $V_{n, r}$ [1974], Stein's generalisations [Brin and Squier 2001; Stein 1992]

This work was partially funded by LMS Scheme 4 grant 41209. The first named author was supported by Gobierno de Aragón, European Regional Development Funds and MTM2015-67781-P (MINECO/FEDER). The second author is a member of the Gruppo Nazionale per le Strutture Algebriche, Geometriche e le loro Applicazioni (GNSAGA) of the Istituto Nazionale di Alta Matematica (INdAM) and gratefully acknowledges the support of the Fondation Mathématique Jacques Hadamard (ANR-10-CAMP-0151-02 - FMJH - Investissement d'Avenir) and the Fundação para a Ciência e a Tecnologia (FCT projects UID/Multi/04621/2013 (CEMAT-Ciências) and PEst-OE/MAT/UI0143/2014). MSC2010: $20 \mathrm{~J} 05$.

Keywords: generalized Thompson groups, finite presentations. 
and Brin's higher dimensional Thompson groups $s V$ [Hennig and Matucci 2012], but not for more complicated generalisations such as the groups $V_{r}(\Sigma)$ we are considering here. These were defined in [Kochloukova et al. 2013; Martínez-Pérez and Nucinkis 2013] and were denoted $G_{r}(\Sigma)$. It is worth pointing out that elements in $V_{r}(\Sigma)$ admit a tree-pair representation similar to that of the original groups $F, T$ and $V$. The authors show in [Martínez-Pérez et al. 2016] that, under some mild hypotheses, being valid and bounded, $V_{r}(\Sigma)$ is the full automorphism group of a Cantor algebra. In the same paper it is shown that under some further minor restrictions, being complete, these groups are of type $\mathrm{F}_{\infty}$ and that this also implies that centralisers of finite subgroups are of type $\mathrm{F}_{\infty}$. We introduce all necessary background in Section 2. The structure of centralisers in $V_{r}(\Sigma)$ is studied in detail in [Martínez-Pérez et al. 2016; Martínez-Pérez and Nucinkis 2013].

One of the objectives of the present paper is to introduce a common framework providing recipes; first to find explicit finite generating sets for the groups $V_{r}(\Sigma)$ in the case when the underlying Cantor algebra $U_{r}(\Sigma)$ is valid and bounded, and second, to find explicit presentations under the additional assumption that $U_{r}(\Sigma)$ is complete. To do that, we construct a model for the classifying space for free actions $\mathrm{E} G$ for $G=V_{r}(\Sigma)$, and use this model to obtain presentations of these groups. As far as we are aware, this construction is new even for the group $V$, and hence could be of independent interest.

In Section 7 we also give an explicit finite presentation for centralisers of finite subgroups for those $V_{r}(\Sigma)$ that are finitely presented. To do so we use the socalled Burnside procedure as used by Guralnick, Kantor, Kassabov and Lubotzky [Guralnick et al. 2011].

In the Appendix we shall give an outline and proof of the Burnside procedure as used in [Guralnick et al. 2011]. This procedure is well known, but we are not aware of any proofs elsewhere. The point is to look for a simple presentation for $G$ that is somehow symmetric and elementary. Initially it may have infinitely many generators and relations; the Burnside procedure offers a way to cut it down to a more manageable, and sometimes finite, presentation.

\section{Background on generalised Thompson groups}

In this section we introduce only those properties of valid bounded Cantor algebras used to make this paper self-contained. For detailed definitions and notation the reader is referred to [Martínez-Pérez and Nucinkis 2013, Section 2], and for proofs of statements cited here, to [Kochloukova et al. 2013; Martínez-Pérez et al. 2016; Martínez-Pérez and Nucinkis 2013].

Let $S=\{1, \ldots, s\}$ be a finite set of colours and associate to each $i \in S$ an integer $n_{i}>1$, called the arity of the colour $i$. For every $i \in S$ consider the following right operations on a set $U$ : 
(i) One $n_{i}$-ary operation $\lambda_{i}: U^{n_{i}} \rightarrow U$, and

(ii) $n_{i}$ unary operations $\alpha_{i}^{1}, \ldots, \alpha_{i}^{n_{i}}$, where $\alpha_{i}^{j}$ is a map $U \rightarrow U$.

We also consider, for each $i \in S$ and $v \in U$, the map

$$
\alpha_{i}: U \rightarrow U^{n_{i}}
$$

given by $v \alpha_{i}:=\left(v \alpha_{i}^{1}, v \alpha_{i}^{2}, \ldots, v \alpha_{i}^{n_{i}}\right)$. The maps $\alpha_{i}$ are called descending operations, or expansions, and the maps $\lambda_{i}$ are called ascending operations, or contractions.

Fix a finite set $X_{r}$ of cardinality $\left|X_{r}\right|=r$. One can define the free object on the set $X_{r}$ with respect to the previous operations which we denote $U$. To define our generalisations of Thompson's group $V$, we will be interested in the free object constructed under the extra requirement that a certain set of laws $\Sigma$ described below must be satisfied. We denote this last free object by $U_{r}(\Sigma)$ and call it the (free) Cantor algebra on $X_{r}$ satisfying $\Sigma$.

Definition 2.1 [Martínez-Pérez and Nucinkis 2013, Section 2]. Fix a finite set $X_{r}$ of cardinality $\left|X_{r}\right|=r$ and consider the free object $U$ on $X_{r}$ with respect to operations (i) and (ii) above. Then $\Sigma=\Sigma_{1} \cup \Sigma_{2}$ with $\Sigma_{1}$ and $\Sigma_{2}$ the following set of laws:

(i) $\Sigma_{1}$ is given by

$$
u \alpha_{i} \lambda_{i}=u, \quad\left(u_{1}, \ldots, u_{n_{i}}\right) \lambda_{i} \alpha_{i}=\left(u_{1}, \ldots, u_{n_{i}}\right),
$$

for every $u \in U, i \in S$, and $n_{i}$-tuple, $\left(u_{1}, \ldots, u_{n_{i}}\right) \in U^{n_{i}}$.

(ii) $\Sigma_{2}$ is given by

$$
\Sigma_{2}=\bigcup_{1 \leq i<i^{\prime} \leq s} \Sigma_{2}^{i, i^{\prime}}
$$

where each $\Sigma_{2}^{i, i^{\prime}}$ is either empty or consists of the following laws: consider first $i$ and fix a map $f:\left\{1, \ldots, n_{i}\right\} \rightarrow\{1, \ldots, s\}$. For each $1 \leq j \leq n_{i}$, we see $\alpha_{i}^{j} \alpha_{f(j)}$ as a set of sequences of length 2 of descending operations and let

$$
\Lambda_{i}=\cup_{j=1}^{n_{i}} \alpha_{i}^{j} \alpha_{f(j)} .
$$

Do the same for $i^{\prime}$ (with a corresponding map $f^{\prime}$ ) to get $\Lambda_{i^{\prime}}$. We need to assume that $f$ and $f^{\prime}$ are chosen so that $\left|\Lambda_{i}\right|=\left|\Lambda_{i^{\prime}}\right|$ and fix a bijection $\phi: \Lambda_{i} \rightarrow \Lambda_{i^{\prime}}$. Then $\Sigma_{2}^{i, i^{\prime}}$ is

$$
u v=u \phi(v), \quad v \in \Lambda_{i}, u \in U .
$$

Let $U_{r}(\Sigma)$ be the algebra obtained from $U$ by quotienting out the relations in $\Sigma$. We say that $U_{r}(\Sigma)$ is valid if for any set $Y \in U$, we have $|Y|=|\bar{Y}|$, where $\bar{Y}$ is the image of $Y$ in $U_{r}(\Sigma)$. In particular this implies that $U_{r}(\Sigma)$ is a free object on $X$ in 
the class of those algebras with the descending and ascending operations (i) and (ii) above which satisfy the identities $\Sigma$.

From now on we work with the free object $U_{r}(\Sigma)$ only. Let $B \subset U_{r}(\Sigma), b \in B$, and let $i$ be a colour of arity $n_{i}$. The set

$$
(B \backslash\{b\}) \cup\left\{b \alpha_{i}^{1}, \ldots, b \alpha_{i}^{n_{i}}\right\}
$$

is called a simple expansion of $B$. Analogously, if $b_{1}, \ldots, b_{n_{i}} \subseteq B$ are pairwise distinct,

$$
\left(B \backslash\left\{b_{1}, \ldots, b_{n_{i}}\right\}\right) \cup\left\{\left(b_{1}, \ldots, b_{n_{i}}\right) \lambda_{i}\right\}
$$

is a simple contraction of $B$. A finite chain of simple expansions is an expansion and a finite chain of simple contractions is a contraction. A subset $A \subseteq U_{r}(\Sigma)$ is called admissible if it can be obtained from the set $X_{r}$ by finitely many expansions or contractions. If a subset $A_{1}$ is obtained from a subset $A$ by an expansion (simple or not), then we write $A \leq A_{1}$.

Remark 2.2. Recall that $U_{r}(\Sigma)$ is said to be bounded (see [Martínez-Pérez and Nucinkis 2013, Definition 2.7]) if for all admissible subsets $Y$ and $Z$ such that there is some admissible $A \leq Y, Z$, there is a unique least upper bound of $Y$ and $Z$. By a unique least upper bound we mean an admissible subset $T$ such that $Y \leq T$ and $Z \leq T$, and whenever there is an admissible set $S$ also satisfying $Y \leq S$ and $Z \leq S$, then $T \leq S$.

By [Kochloukova et al. 2013, Lemma 2.5], any admissible set is a basis of $U_{r}(\Sigma)$. Conversely, by [Martínez-Pérez et al. 2016, Theorem 2.5], if $\Sigma$ is valid and bounded, any basis of $U_{r}(\Sigma)$ is also an admissible set. Furthermore, for every admissible subset of cardinality $m$, we have that

$$
m \equiv r \bmod d \quad \text { for } d:=\operatorname{gcd}\left\{n_{i}-1 \mid i=1, \ldots, s\right\} .
$$

In particular, any basis with $m$ elements can be transformed into one of $r$ elements. Hence $U_{r}(\Sigma)=U_{m}(\Sigma)$ and we may assume that $r \leq d$.

Definition 2.3. [Martínez-Pérez and Nucinkis 2013, Definition 2.12] Let $U_{r}(\Sigma)$ be a valid Cantor algebra. We denote the group of all Cantor algebra automorphisms of $U_{r}(\Sigma)$ by $V_{r}(\Sigma)$. In particular, these automorphisms are induced by a map $V \rightarrow W$, where $V$ and $W$ are admissible subsets of $U_{r}(\Sigma)$ of the same cardinality. In our notation automorphisms act on the left.

For example, when $s=1$ we have $\Sigma_{2}=\varnothing$ and we retrieve the original HigmanThompson groups $G_{r, n}$ (here, $n=n_{1}$ ) [Higman 1974]. For

$$
s=2, \quad r=1 \quad \text { and } \quad n_{1}=n_{2}=2,
$$


the Brin-Thompson groups are now given by the set $\Sigma_{2}$ that can be visualised as follows:
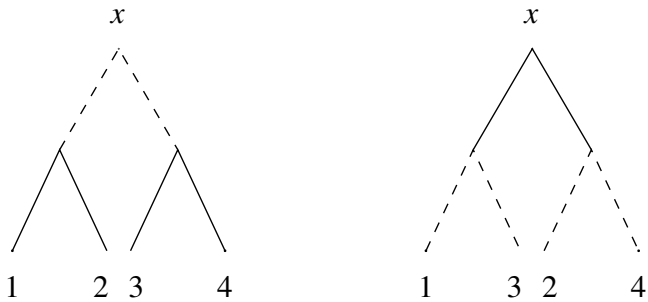

Here dashed and solid lines represent expansions of different colours. For more examples the reader is referred to [Martínez-Pérez et al. 2016; Martínez-Pérez and Nucinkis 2013].

Remark 2.4. If $U_{r}(\Sigma)$ is valid and bounded every element of $V_{r}(\Sigma)$ can be given by a bijection $V \rightarrow W$, where $V$ and $W$ are descendants of the fixed basis $X_{r}$.

For $r=1$, this means that we can visualise elements of $V_{1}(\Sigma)$ by tree-pair diagrams of rooted trees, where the root represents the basis $X_{1}=\{x\}$. So, for example, when $s=1$ and $n_{1}=2, V_{1}(\Sigma)$ is equal to $V$ (the original Thompson group), and the well-known generator $x_{0} \in F \subset V$ is visualised as
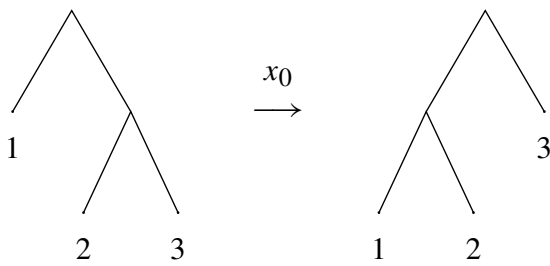

Definition 2.5 [Martínez-Pérez et al. 2016, Definition 3.2]. Let $B \leq A$ be admissible subsets of $U_{r}(\Sigma)$. We say that the expansion $B \leq A$ is elementary if there are no repeated colours in the paths from elements in $B$ to their descendants in $A$. We denote an elementary expansion by $B \preceq A$. We say that the expansion is very elementary if all paths have length at most 1.

Denote by $\mathcal{P}_{r}$ the poset of admissible subsets in $U_{r}(\Sigma)$, and by $\left|\mathcal{P}_{r}\right|$ its geometric realisation. (It was shown in [Martínez-Pérez and Nucinkis 2013] that $\left|\mathcal{P}_{r}\right|$ is a model for $\underline{E} G$, the classifying space for proper actions). We now describe the Stein complex $\mathcal{S}_{r}(\Sigma)$ [Stein 1992], which is a subcomplex of $\left|\mathcal{P}_{r}\right|$. The vertices in $\mathcal{S}_{r}(\Sigma)$ are given by the admissible subsets of $U_{r}(\Sigma)$. The $k$-simplices are given by chains of expansions $Y_{0} \leq \cdots \leq Y_{k}$, where $Y_{0} \preceq Y_{k}$ is an elementary expansion.

From now on we will denote $V_{r}(\Sigma)$ by $G$. In the next section we will use $\mathcal{S}_{r}(\Sigma)$ to construct a model for E $G$. Recall that by [Martínez-Pérez et al. 2016, Lemma 3.6 and Remark 3.7], $\mathcal{S}_{r}(\Sigma)$ is contractible and has finite stabilisers. 


\section{A model for $\mathbf{E} G$}

In this section we construct a model for the space $\mathrm{E} G$ when $G$ is the automorphism group of a valid and bounded Cantor algebra $U_{r}(\Sigma)$ as before. We shall use this model to get, initially infinite, presentations for our groups, which we will then reduce to obtain a finite generating set, and later a finite presentation under some extra hypothesis on $U_{r}(\Sigma)$.

3A. Some technical observations. To begin with, we collect few technical observations that we will use later on. As seen before, the elements $g$ in our group $G$ can be expressed via a bijection between a pair of admissible subsets (or bases) $\left(B, B^{\prime}\right)$ both of the same cardinality. Observe that the pair above is not enough to determine $g$ and that we have to specify the explicit bijection. A way to overcome this problem is to work with ordered bases, in the sense that instead of a basis $B$ viewed as a set, we will be considering an ordered tuple $A$ with underlying set $B$. We say $u(A)=B$ ( $u$ for underlying). A pair of ordered tuples $\left(A, A^{\prime}\right)$, with both $A$ and $A^{\prime}$ of the same cardinality, uniquely determines the element of $G$ mapping the elements of $A$ to the elements of $A^{\prime}$ in the prescribed order; conversely, any group element is expressible in this way. Of course, just as for the representation of the pair of bases, there is not a unique pair $\left(A, A^{\prime}\right)$ determining a given $g \in G$ : we may apply descending or ascending operations to $A$ and $A^{\prime}$ in a consistent way to get a new pair of ordered tuples representing the same group element. Moreover, we may also permute the elements of both tuples in a consistent way and still get the same $g$. This means that when we represent elements of $G$ as pairs $\left(A, A^{\prime}\right)$ we should be talking about equivalence classes of pairs under the obvious equivalence relation that identifies pairs yielding the same element. However, to make the notation lighter we will talk about pairs and denote them as above. The following definition will be useful later on: given tuples $A_{1}, A_{2}$ with bases as underlying sets we put

$$
A_{1} \precsim A_{2} \Longleftrightarrow u\left(A_{1}\right) \leq u\left(A_{2}\right) \text { is an elementary expansion. }
$$

Equivalently, $A_{1} \precsim A_{2}$ if $u\left(A_{1}\right) \leq u\left(A_{2}\right)$ in the Stein poset. Observe that this is not a partial order, as it is not antisymmetric: we could have $A_{1} \precsim A_{2}$ and $A_{2} \precsim A_{1}$ but $A_{1} \neq A_{2}$. When $A_{1} \precsim A_{2}$, abusing the terminology slightly, we will say that $A_{2}$ is obtained from $A_{1}$ by descending operations. Essentially this means that we are considering the permutation of the elements of a tuple as a new type of descending operation. Of course this could equally be viewed as an "ascending" operation, but it turns out to be convenient to view it as descending. If we want to record the precise operations that yield $A_{2}$ when applied to $A_{1}$ we will write

$$
A_{1} \stackrel{\varepsilon}{\precsim} A_{2}
$$

and will also set $A_{2}=A_{1} \varepsilon$. Observe that $\varepsilon$ can be seen as a precise recipe to get $A_{2}$, and $\varepsilon$ encodes exactly which elements are modified, permuted and so on. 
3B. The model for $\boldsymbol{E} \boldsymbol{G}$. Let $Z$ be the complex constructed as follows: The points of $Z$ are the ordered tuples $A$ with underlying set a basis $u(A)$ in the Stein complex $\mathcal{S}_{r}(\Sigma)$. For each chain

$$
A_{0} \precsim \cdots \precsim A_{k}
$$

we attach an (oriented) $k$-simplex at the vertices $A_{0}, \ldots, A_{k}$. Observe that there might be repeated vertices, so this is not a simplicial complex but rather has the structure of a $\Delta$-complex; see [Hatcher 2002, Section 2.1]. The group $G$ acts on the set of bases, and using that action one can define a $G$-action on $Z$ in the obvious way. Note that this action is free. In particular this implies that two different 1 -simplices starting in $A_{0}$, say $A_{0} \precsim A_{1}$ and $A_{0} \precsim A_{1}^{\prime}$ cannot be in the same $G$-orbit. Hence they yield different 1 -simplices in the quotient complex $Z / G$. Conversely, if $\bar{A}_{0} \stackrel{\bar{\varepsilon}}{\rightarrow} \bar{A}_{1}$ is an edge in $Z / G$, then once we have fixed a lift $A_{0}$ of $\bar{A}_{0}$ to $Z, \bar{\varepsilon}$ lifts to a unique 1-simplex of $Z$. Therefore there is some well-defined set of descending operations giving a tuple $A_{1}^{\prime}$ which is uniquely determined so that $A_{0} \underset{\varepsilon}{\precsim} A_{1}^{\prime}$ is the lift of $\bar{\varepsilon}$. Moreover, the tuple $A_{1}^{\prime}$ is uniquely determined. Note that we have the extra restriction coming from the Stein poset: we can only apply descending operations of the same colour once to any element of $A_{0}$.

Applying the same argument implies that this also holds for any lift of a path in $Z / G$ to $Z$.

We now show that $Z$ is contractible by using the contractibility of $\mathcal{S}_{r}(\Sigma)$, [Stein 1992]. There is a $G$-map

$$
u: Z \rightarrow \mathcal{S}_{r}(\Sigma)
$$

associating the underlying basis to an ordered tuple.

Fix a basis $B \in \mathcal{S}_{r}(\Sigma)$ of cardinality $k$. Then $u^{-1}(B)$ is the full subcomplex of $Z$ with 0 -simplices given by the tuples with underlying set $B$, i.e., given by all possible permutations of the elements in $B$. Let $H$ the stabiliser of $B$ in $G$. Then $H$ is isomorphic to the symmetric group of degree $k$ and acts freely on the 0 -simplices of $u^{-1}(B)$. In fact we may choose a bijection between the 0 -simplices of $u^{-1}(B)$ and the elements of $H$ and the definition of the complex structure of $Z$ means that any $(k+1)$-element subset of 0 -simplices spans a $k$-simplex.

For example if $H=S_{2}$ is the symmetric group on two letters with elements 1 and $x$, then the 1 -simplices are $\{1,1\},\{1, x\},\{x, 1\}$ and $\{x, x\}$, and the 2-simplices are $\{1,1,1\},\{1,1, x\}$ etc.

In other words, $u^{-1}(B)$ is easily seen to be the usual complex associated to the bar resolution of the finite group $H$; see for example [Hatcher 2002, Example 1B.7]. In particular this shows that $u^{-1}(B)$ is contractible.

Using [Quillen 1973, Theorem A], we can now show that $u$ is a homotopy equivalence. To see this, let $J_{Z}$ be the category with objects the simplices of $Z$ and morphisms given by the face relations. Note that since $Z$ is not a simplicial 
complex, this is not a poset. Let $J_{S}$ be the poset of simplices in $\mathcal{S}_{r}(\Sigma)$. The map $u$ induces a functor

$$
J_{u}: J_{Z} \rightarrow J_{S},
$$

and the geometric realisations of nerves of the categories $J_{Z}$ and $J_{S}$ are the barycentric subdivisions of $Z$ and $\mathcal{S}_{r}(\Sigma)$, respectively. Once we show that for any $\sigma: B_{0}<B_{1}<\cdots<B_{t}$ in $J_{S}$,

$$
J_{u} / \sigma:=\left\{\tau \in J_{Z} \mid J_{u}(\tau) \text { is a subsimplex of } \sigma\right\}
$$

is a contractible subcategory of $J_{Z}$, we can use Quillen's Theorem A to deduce that $J_{u}$ is a homotopy equivalence. The category $J_{u} / \sigma$ is just the category with objects the simplices in the join

$$
u^{-1}\left(B_{0}\right) \star \cdots \star u^{-1}\left(B_{t}\right)
$$

and morphisms given by face relations. As $u^{-1}\left(B_{0}\right) \star \cdots \star u^{-1}\left(B_{t}\right)$ is contractible, this category is also contractible. Hence $J_{u}$ is a homotopy equivalence and thus $u$ is, too. Since $\mathcal{S}_{r}(\Sigma)$ is contractible we deduce that $Z$ is contractible as required.

\section{An infinite presentation}

In this section we use the model for $\mathrm{E} G$ that we have just constructed to obtain a presentation for our group. As the model is of infinite type, our presentation will initially be infinite. But in the case when the Cantor algebra is valid and bounded it is possible to reduce the generating system to a finite one, as we will see in the next section.

We obtain our presentation using the following well known result that we recall here for the reader's convenience.

Theorem 4.1 [Geoghegan 2008, Theorem 3.1.16 and Corollary 3.1.17]. Let $G$ be a group and $Z$ a simply connected $C W$-complex with a free $G$-action such that $Z / G$ is oriented and path connected. Let $\mathcal{T}$ be a maximal tree in $Z / G$. Let:

- $W$ be the set of (oriented) 1-cells of $Z / G$.

- $R$ be the set of words in the alphabet $W \cup W^{-1}$ obtained as follows: for each (oriented) 2-cell $e_{\gamma}^{2}$ in $Z / G$, let $\tau\left(e_{\gamma}^{2}\right)$ be a word representing the boundary $\delta e_{\gamma}^{2}$ and set

$$
R=\left\{\tau\left(e_{\gamma}^{2}\right) \mid e_{\gamma}^{2} \text { is an oriented 2-cell of } Z / G\right\} .
$$

- $S \subset W$ be the set of (oriented) 1-cells of $\mathcal{T}$ (seen as one letter words in $W$ ).

Then

$$
\langle W \mid R \cup S\rangle
$$

is a presentation of the group $G \cong \pi_{1}(Z / G)$. If, moreover, $Z / G$ has a finite 2-skeleton, then this is a finite presentation. 
4A. The isomorphism $G \cong \pi_{1}(Z / G)$. We now give an explicit isomorphism between $G$ and the fundamental group of $Z / G$, where we return to our previous notation so $G=V_{r}(\Sigma)$ and $Z$ is the same complex as in Section 3B. The standard way to show this isomorphism is to fix some point $x_{0} \in Z$ and map the element $g \in G$ to the path in $Z / G$ obtained by taking the quotient of a path from $x_{0}$ to $g x_{0}$ in $Z$. As $x_{0}$ and $g x_{0}$ have the same cardinality, what we get is a loop path in $Z / G$. We shall take as $x_{0}$ a tuple with underlying set our preferred basis of $r$ elements $X_{r}$. To ease notation, we denote this tuple by $X_{r}$ as well. As the $G$-action on $Z$ preserves the cardinality of each tuple, the 0 -simplices of $Z / G$ correspond to the possible cardinalities of tuples (or of bases). By Remark 2.2, we recall that the possible cardinalities of the bases are exactly the integers congruent to $r$ modulo $d$ where $n_{1}, \ldots, n_{s}$ are the arities and

$$
d=\operatorname{gcd}\left(n_{1}-1, \ldots, n_{s}-1\right) .
$$

So the 0 -simplices of $Z / G$ can be labelled as

$$
\left\{\bar{X}_{i} \mid i \equiv r \bmod d\right\}
$$

where the subscript is the cardinality of the associated bases. Now, choose a maximal tree $\mathcal{T}$ in $Z / G$. The vertices of $\mathcal{T}$ are all the 0 -simplices above and there is a unique path in $\mathcal{T}$ from $\bar{X}_{r}$ to every other $\bar{X}_{i}$. This path determines uniquely a precise tuple $X_{i}$ that is a lift of $\bar{X}_{i}$ (observe that $X_{i}$ depends on the choice of $\mathcal{T}$ ).

Let $\bar{X}_{i} \stackrel{\bar{\varepsilon}}{\rightarrow} \bar{X}_{j}$ be an edge (thus $i \leq j$ ). By the comments above there is a uniquely determined lift $X_{i} \stackrel{\varepsilon}{\rightarrow} X_{j}^{\prime}$ of $\bar{\varepsilon}$; here $X_{j}^{\prime}$ is a new tuple which is in the same orbit as $X_{j}$. Therefore there is a uniquely determined $g \in G$ such that $X_{j}^{\prime}=g X_{j}$, and this is precisely the element in $G$ corresponding to the generator

$$
\bar{\varepsilon} \in \pi_{1}(Z / G) .
$$

We have $g=\left(X_{j}, X_{j}^{\prime}\right)$ and $X_{j}^{\prime}=X_{i} \varepsilon$.

Example 4.2. Let $G$ be the original Thompson group $V$. In particular, $r=1, s=1$, $n_{1}=2$. We can represent bases of $U_{1}(\Sigma)$ by finite rooted binary trees, and hence can choose $X_{1}$ to be a single point, and $X_{2}$ and $X_{3}$ to be the bases represented thus:
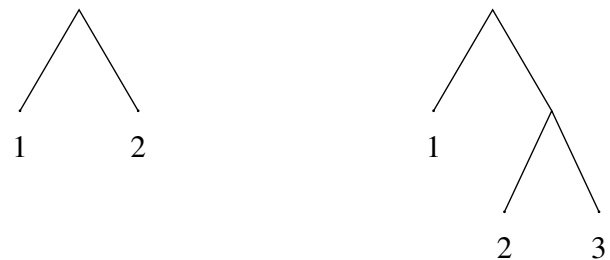

$X_{2}$

$X_{3}$ 
Suppose we take $\varepsilon$ to be the expansion of $X_{2}$ on the left-hand leaf. This gives us $X_{3}^{\prime}$ as

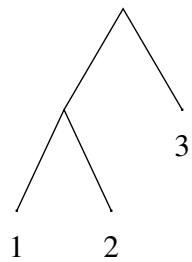

and the corresponding element of $V$ is $x_{0}$ as described after Remark 2.4.

4B. The maximal tree $\mathcal{T}$. To be able to write down an explicit presentation, the choice for $\mathcal{T}$ becomes important. This relies heavily on the choice of representative for $\overline{X_{i}}$ above. This amounts to choosing a particular set of bases $X_{i}$ in $U_{r}(\Sigma)$, where $i \equiv r \bmod d$.

Example 4.3. For $G=s V$ we have $r=1$ and for each $k \in \mathbb{N}$ there is a basis $X_{k}$. Again, these can be represented by finite rooted binary trees. Now fix a colour $i \in S$ and choose the $X_{k}$ as follows: we begin with $X_{1}$ our fixed one-element basis represented by a single point. Now $X_{2}$ is the basis obtained by applying the descending operation of colour $i$ to $X_{1}$. We successively chose $X_{k}$ as obtained from $X_{k-1}$ by applying the descending operation of colour $i$ to the last element of $X_{k-1}$ and labelling the elements in successive order. The representation for $X_{k}$ by a binary tree then looks as follows:

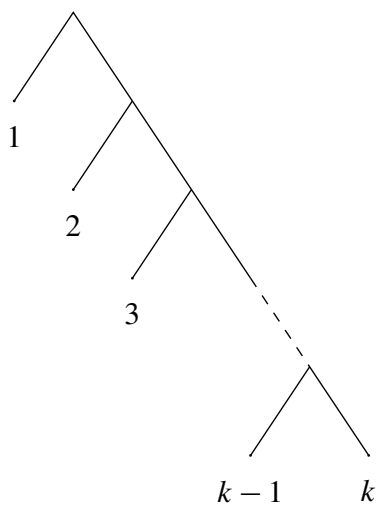

Notice that for $V_{1}(\Sigma)$ we can always choose the $X_{k}$ to be represented by a rightmost tree as above, provided that all colours have the same arity. Now the construction shows that the maximal tree $\mathcal{T}$ in $Z / G$ is a rooted infinite line.

For example, the baker's map $b \in 2 V$ can easily be described using the bases chosen above. Let $X_{1}$ be a single point and $X_{2}$ be as in Example 4.2; note that we expanded with colour 1 . Now we consider $X_{2}^{\prime}$ the basis obtained from $X_{1}$ by 
expanding once with colour 2 (represented by a dashed line). Hence this gives rise to the element $b \in 2 V$.
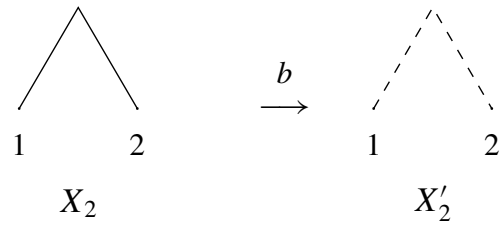

For the general case with mixed arities we will not be able to find such a straightforward set of representatives $X_{i}$ as before. We will show that we can, however, find a maximal tree $\mathcal{T}$ in $Z / G$, whose vertices are all but a finite number obtained by a step-by-step process beginning with our fixed basis $X_{r}$ and then expanding the last element of a basis previously constructed.

Example 4.4. Let $V_{r}(\Sigma)$ be the group given by $r=1, s=2, n_{1}=5$ and $n_{2}=7$. Then $d=2$ and our chosen set of bases is of the form

$$
\left\{X_{i} \mid i \equiv 1 \bmod 2\right\}
$$

By simply expanding $X_{1}$ by the colours 1 and 2 respectively, we obtain $X_{5}$ and $X_{7}$. To obtain $X_{3}$ we could contract the last 5 elements of $X_{7}$ by colour 1 , but there is no way to obtain $X_{3}$ from $X_{1}$ by simply expanding.

Remark 4.5. We now describe the construction of our preferred maximal tree $\mathcal{T}$ in $Z / G$, where $G=V_{r}(\Sigma)$ is the automorphism group of a valid and bounded Cantor algebra. We begin by showing that we can obtain all but finitely many of the bases

$$
\left\{X_{i} \mid i \equiv r \bmod d\right\}
$$

from $X_{r}$ applying descending operations only. In other words

$$
\left\{r+\sum_{i=1}^{s} k_{i}\left(n_{i}-1\right) \mid 0 \leq k_{1}, \ldots, k_{s}\right\} \cup P=\{r+k d \mid 0 \leq k\},
$$

where $P$ is a finite set of integers. To see this, observe first that the problem can be reduced to the case when $r=0$ and $d=1$. Now choose integers $k_{1}, \ldots, k_{s}$ such that

$$
1=\sum_{i=1}^{s} k_{i}\left(n_{i}-1\right)
$$

and use them to produce integers $m_{1}, \ldots, m_{s}$ with $0 \leq m_{2}, \ldots, m_{s}$ such that

$$
1=\sum_{i=1}^{s} m_{i}\left(n_{i}-1\right)
$$


Hence,

$$
1 \equiv \sum_{i=2}^{s} m_{i}\left(n_{i}-1\right) \bmod m_{1} .
$$

Multiplying this expression by the integers $2, \ldots, m_{1}$ we get positive numbers $a_{1}, \ldots, a_{m_{1}}$ which are a complete set of representatives of the residues modulo $m_{1}$ and such that they all belong to $\sum_{i=1}^{s} \mathbb{N}\left(n_{i}-1\right)$. Now, let $m$ be any integer with $m \geq \max \left\{a_{i} \mid 1 \leq i \leq m_{1}\right\}$. Then for some such $i$, we have $m \equiv a_{i}$ modulo $m_{1}$ and therefore $m-a_{i}=l m_{1}$ for some $l \geq 0$. From this we deduce that $m$ also belongs to $\sum_{i=1}^{s} \mathbb{N}\left(n_{i}-1\right)$.

It is now easy to find a (nonmaximal) directed tree in $Z / G$ having $\bar{X}_{r}$ as a root and such that the cardinalities of the vertices are precisely the set $r+\sum_{i=1}^{s} \mathbb{N}\left(n_{i}-1\right)$. Here, a root is the only vertex of the tree from which all other vertices can be reached by paths respecting the directions of the edges. Moreover, we can do it in such a way that the descending operations are always applied to the last element of each tuple. There are only finitely many points of our space $Z / G$ not in this tree. Choose one of them and consider a directed path from that point to some point of the tree. Adding this directed path we get a new tree which no longer has a single root in the above sense. If there are still points left, repeat the process. Eventually, we get a directed tree with the desired properties and with only finitely many roots.

4C. The presentation. Now we apply Theorem 4.1 to produce an explicit presentation. We do get an abstract group presentation but we can also write it down as a presentation in terms of elements given by pairs of ordered bases using the explicit isomorphism in Section 4A, which allows one to recognise the group elements in a much more familiar way. Recall that we have fixed a set of tuples

$$
\left\{X_{i} \mid i \equiv r \bmod d\right\}
$$

which are lifts of the nodes of our tree $\mathcal{T}$. Moreover there is a tree in $Z$ that is a lift of $\mathcal{T}$.

By [Geoghegan 2008, Theorem 3.1.16], $\pi_{1}(Z / G)$ is generated by the edges in $Z / G$, i.e., by the 1 -simplices $\bar{X}_{i} \stackrel{\bar{\varepsilon}}{\rightarrow} \bar{X}_{j}$ in $Z / G$. As we have seen before, these correspond to elements $g \in G$ which are given by pairs $\left(X_{j}, X_{i} \varepsilon\right)$ where $\varepsilon$ is a set of descending operations.

There are two sets of relators:

(i) Relators of the form $\bar{\varepsilon}=1$ whenever $\bar{\varepsilon}$ is an edge in the tree $\mathcal{T}$. This means that there are tuples $X_{i}$ and $X_{j}$ in $\mathcal{T}$ such that $X_{j}$ is obtained from $X_{i}$ performing the operations $\varepsilon$. The group element that corresponds to $\varepsilon$ is $\left(X_{j}, X_{j}\right)$.

(ii) Relators obtained from the boundaries of the 2-cells in $Z / G$. The 2-cells of $Z / G$ come from 2-cells in $Z$ and these are of the form $A_{0} \precsim A_{1} \precsim A_{2}$. Let $\varepsilon_{1}$ 
be the set of operations needed to obtain $A_{1}$ from $A_{0}, \varepsilon_{2}$ the set of operations needed to obtain $A_{2}$ from $A_{1}$ and $\varepsilon$ the composition of $\varepsilon_{1}$ and $\varepsilon_{2}$. Passing down to the quotient $Z / G$ we get a 2-cell with boundary labelled $\bar{\varepsilon}_{1}, \bar{\varepsilon}_{2}$ and $\bar{\varepsilon}$. So we have the relator

$$
\bar{\varepsilon}=\bar{\varepsilon}_{1} \bar{\varepsilon}_{2}
$$

All this means that this second set of relators consists of the "composition of paths". We want to write this down in terms of pairs of ordered bases. Let $i$ be the cardinality of $A_{0}$ and $j_{1}$ the cardinality of $A_{1}$. The edge $\bar{\varepsilon}_{1}$ represents the element $g_{1}=\left(X_{j_{1}}, X_{i} \varepsilon_{1}\right) \in G$. We may apply the descending operations $\varepsilon_{2}$ to this pair and then we observe that also $g_{1}=\left(X_{j_{1}} \varepsilon_{2}, X_{i} \varepsilon_{1} \varepsilon_{2}\right)$. Note here that this follows from the definition of tree pair representation, and we do not need to impose any conditions on the presentation that we are building. Let $j_{2}$ be the cardinality of $A_{2}$, then $\bar{\varepsilon}_{2}$ represents the element $g_{2}=\left(X_{j_{2}}, X_{j_{1}} \varepsilon_{2}\right)$ and $\bar{\varepsilon}$ represents $g=\left(X_{j_{2}}, X_{i} \varepsilon\right)$. So we get the relator $g=g_{1} g_{2}$. In the particular case when $X_{j_{1}} \varepsilon_{2}$ belongs to the lift of our tree $\mathcal{T}$, or equivalently when $\bar{\varepsilon}_{2}$ belongs to $\mathcal{T}$, there is also a relator $g_{2}=1$ and we deduce $g=g_{1}$. This can also be seen using tree pairs: as $X_{j_{2}}$ belongs to the prefixed set of nodes and has the same cardinality as $X_{j_{1}} \varepsilon_{2}$, we must have $X_{j_{1}} \varepsilon_{2}=X_{j_{2}}$.

We may summarise as follows:

$$
G=\langle W \mid R\rangle,
$$

where

$$
W=\left\{\left(X_{j}, X_{i} \varepsilon\right) \mid \varepsilon \text { is a sequence of descending operations and } X_{i} \neq X_{j}\right\},
$$

$$
R=\left\{g=g_{2} g_{1} \mid g=\left(X_{j_{2}}, X_{i} \varepsilon\right), g_{1}=\left(X_{j_{1}}, X_{i} \varepsilon_{1}\right), g_{2}=\left(X_{j_{2}}, X_{j_{1}} \varepsilon_{2}\right), \varepsilon=\varepsilon_{1} \varepsilon_{2}\right\} .
$$

Alternatively, we may delete those pairs $\left(X_{j}, X_{i} \varepsilon\right)$ where $\bar{\varepsilon}$ lies in the tree $\mathcal{T}$ from our list of generators.

4D. Reducing the generating set. A quick look to the generating set we have just obtained shows that it is far too big. Reducing it can be a complicated task but there is a reduction that seems natural: our generators come from edges in $Z / G$ and these edges come from descending operations, so one expects that edges coming from "very elementary operations" should be enough. This is in fact the case but to make it more precise we need now some additional technicalities. Let us fix what should be called "very elementary" in our context. An edge $A_{1} \stackrel{\varepsilon}{\precsim} A_{2}$ in $Z$ is very elementary if it consists of a single operation, i.e., if it is either a permutation or it is a single descending operation (in this case $u\left(A_{1}\right)<u\left(A_{2}\right)$ is very elementary) but we do not allow composition of both. The case when $u\left(A_{1}\right)<u\left(A_{2}\right)$ will be termed strict and for these type of operations we will assume that if the original 
tuple is $\left(x_{1}, \ldots, x_{i}\right)$ and we apply the descending operation $\alpha$ at the $k$-th element then the resulting tuple is

$$
\left(x_{1}, \ldots, x_{k-1}, x_{k} \alpha^{1}, \ldots, x_{k} \alpha^{n_{\alpha}}, x_{k+1}, \ldots, x_{i}\right) .
$$

Any $\varepsilon$ can be written as a composition of very elementary operations. Of course it may happen that different sequences of operations give the same result when applied to the same tuple. This happens in the following four ways, which we shall refer to as moves:

(i) Disjoint type: we may apply two very elementary strict descending operations acting on distinct elements of a tuple and we get the same result regardless of the order of application of these two operations.

(ii) $\Sigma$ type: we have different chains of elementary strict descending operations such that, up to a permutation, they give the same result when applied to any element of any tuple and which come from the defining relations for the algebra encoded in $\Sigma$.

(iii) Permutation-descending: we may first permute the elements of a tuple and then apply a very elementary strict descending operation or do it the other way around in a consistent manner and get the same result.

(iv) Permutation: the composition of two permutations is still a permutation.

Lemma 4.6. Let $A_{1}, A_{2}$ be tuples. If two different chains of very elementary descending operations yield $A_{2}$ when applied to $A_{1}$, then one can be obtained from the other by repeated application of moves of the four types above.

Proof. By making moves of types (iii) and (iv) only we may assume that our two chains are of the form

$$
\begin{aligned}
& \varepsilon_{1} \varepsilon_{2} \cdots \varepsilon_{t} \sigma, \\
& \varepsilon_{1}^{\prime} \varepsilon_{2}^{\prime} \cdots \varepsilon_{t^{\prime}}^{\prime} \sigma^{\prime},
\end{aligned}
$$

where all $\varepsilon_{i}, \varepsilon_{i}^{\prime}$ are very elementary and strict and $\sigma, \sigma^{\prime}$ are permutations. Consider first what happens when we look at the underlying sets $u\left(A_{1}\right)$ and $u\left(A_{2}\right)$. The fact that both series of operations give the same set when applied to $u\left(A_{1}\right)$, implies that, for each particular element, we are either performing the same operation or the same operation up to applying some of the relators encoded in $\Sigma$. This means that $\varepsilon_{1} \varepsilon_{2} \cdots \varepsilon_{t}$ can be transformed to $\varepsilon_{1}^{\prime} \varepsilon_{2}^{\prime} \cdots \varepsilon_{t^{\prime}}^{\prime}$ by making moves of types (i) or (ii) without taking the order of the elements into account. The fact that the relations in $\Sigma$ involve certain permutations implies that what we really get is that via some extra moves of types (iii) and (iv), $\varepsilon_{1} \varepsilon_{2} \cdots \varepsilon_{t}$ is transformed to $\varepsilon_{1}^{\prime} \varepsilon_{2}^{\prime} \cdots \varepsilon_{t^{\prime}}^{\prime} \tau$ for a certain permutation $\tau$. So at this point our two sequences are

$$
\varepsilon_{1}^{\prime} \varepsilon_{2}^{\prime} \cdots \varepsilon_{t^{\prime}}^{\prime} \tau \sigma, \quad \varepsilon_{1}^{\prime} \varepsilon_{2}^{\prime} \cdots \varepsilon_{t^{\prime}}^{\prime} \sigma^{\prime}
$$


The fact that both sequences yield $A_{2}$ when applied to $A_{1}$ implies that $B \tau \sigma=B \sigma^{\prime}$ for $B=A_{1} \varepsilon_{1}^{\prime} \varepsilon_{2}^{\prime} \cdots \varepsilon_{t^{\prime}}^{\prime}$, which is a move of type (iv).

We next use Tietze transformations to change the presentation above. Essentially, what we need to do is the following: whenever there is a relator $g=g_{1} g_{2}$ we delete $g$ from our set of generators. The effect of this transformation on the generating set is that we no longer have elements $g$ coming from edges which are not very elementary. Moreover we will have only two kinds of generators: strict generators coming from strict very elementary edges, and finite order generators coming from permutations. We denote these sets by

$$
W_{s}=\left\{\left(X_{j}, X_{i} \varepsilon\right) \mid \varepsilon \text { is a very elementary strict expansion, } j=\left|X_{i} \varepsilon\right|\right\}
$$

and call these very elementary strict generators. We also consider the elements of the set

$$
W_{p}=\left\{\left(X_{i}, X_{i} \sigma\right) \mid \sigma \text { is a permutation }\right\},
$$

and call them permutations. From now on we will use the term strict generators for elements in $W_{s}$ instead of the more precise very elementary strict generator.

The effect of this transformation on the set of relators is as follows: we no longer have to consider relators coming from edges in the tree. Whenever there are two sequences of very elementary operations that give the same $A_{2}$ when applied to some $A_{1}$, we have a new relator. Lemma 4.6 implies that these relators can be obtained from relators of the following types:

(i) $R_{D}$ contains relators of the form $g_{1} g_{2}=g_{2}^{\prime} g_{1}^{\prime}$ with $g_{1}, g_{2}, g_{1}^{\prime}, g_{2}^{\prime}$ strict generators coming from moves of disjoint type.

(ii) $R_{\Sigma}$ contains relators between strict generators possibly followed by a permutation coming from moves of $\Sigma$ type.

(iii) $R_{P D}$ contains relators of the form $g \sigma=\sigma g$ with $g$ a strict generator and $\sigma$ a permutation coming from moves of type (iii).

(iv) $R_{P}$ contains relators of the form $\sigma=\sigma_{1} \sigma_{2}$ with $\sigma, \sigma_{1}$ and $\sigma_{2}$ permutations coming from moves of type (iv).

Thus $G$ admits the following (infinite) presentation:

$$
\left\langle W_{s} \cup W_{p} \mid R_{D} \cup R_{\Sigma} \cup R_{P D} \cup R_{P}\right\rangle .
$$

4E. Being more explicit. Let us consider an arbitrary strict generator $\left(X_{j}, X_{i} \varepsilon\right)$ associated to the strict edge $\bar{\varepsilon}$. It is completely determined by a triple $(i, k, t)$ meaning that $\bar{\varepsilon}$ is obtained by applying the descending operation of colour $t$ to the $k$-th element of an orbit representative of the set of tuples of order $i$. We will use the triple to denote the generator. Now we are going to write down explicitly what relators of disjoint type look like with this new notation. Recall that these relators 
come from very elementary strict descending and disjoint operations $\varepsilon_{1}, \varepsilon_{2}$ on one hand, and $\varepsilon_{2}^{\prime}, \varepsilon_{1}^{\prime}$ on the other. They are such that

$$
\varepsilon_{1} \varepsilon_{2}=\varepsilon_{2}^{\prime} \varepsilon_{1}^{\prime},
$$

where $\varepsilon_{1}$ and $\varepsilon_{1}^{\prime}$ are operations of the same colour, say $t$, whereas $\varepsilon_{2}$ and $\varepsilon_{2}^{\prime}$ are of colour $s$. Moreover $\varepsilon_{1}$ acts at the $k_{1}$-th and $\varepsilon_{2}^{\prime}$ acts at the $k_{2}$-th elements of $X_{i}$. We may assume that $k_{1}<k_{2}$. Observe that this means that if we apply a descending operation to the $k_{2}$-th element first then the $k_{1}$-th element remains the same, but if we do it the other way around, i.e., apply a descending operation of colour $t$ to the $k_{1}$-th element first, then the former $k_{2}$-th element becomes the $\left(k_{2}+n_{t}-1\right)$-th. Therefore the triples associated to each of $\bar{\varepsilon}_{1}, \bar{\varepsilon}_{2}, \bar{\varepsilon}_{2}^{\prime}, \bar{\varepsilon}_{1}^{\prime}$ are

$$
\begin{aligned}
& \bar{\varepsilon}_{1}:\left(i, k_{1}, t\right)=\left(X_{i+n_{t}-1}, X_{i} \varepsilon_{1}\right), \\
& \bar{\varepsilon}_{2}:\left(i+n_{t}-1, k_{2}+n_{t}-1, s\right)=\left(X_{i+n_{t}-1+n_{s}-1}, X_{i+n_{t}-1} \varepsilon_{2}\right), \\
& \bar{\varepsilon}_{2}^{\prime}:\left(i, k_{2}, s\right)=\left(X_{i+n_{s}-1}, X_{i} \varepsilon_{2}^{\prime}\right), \\
& \bar{\varepsilon}_{1}^{\prime}:\left(i+n_{s}-1, k_{1}, t\right)=\left(X_{i+n_{s}-1+n_{t}-1}, X_{i+n_{s}-1} \varepsilon_{1}\right),
\end{aligned}
$$

and our relator is

$$
\left(i, k_{1}, t\right)\left(i+n_{t}-1, k_{2}+n_{t}-1, s\right)=\left(i, k_{2}, s\right)\left(i+n_{s}-1, k_{1}, t\right) .
$$

Analogously, it is possible to represent a generator $\left(X_{i}, \sigma\left(X_{i}\right)\right)$ of "permutation type" using the pair $(i, \sigma)$. Now, relators of type $R_{P D}$ come from the fact that applying first a permutation and then a very elementary strict operation to a tuple, yields the same as doing it the other way around for a suitable permutation. More explicitly, assume that we start with the tuple $X_{i}$. Let $\varepsilon$ be the operation associated to the triple, say, $(i, k, t)$ and consider a permutation $\sigma$ represented by the pair $(i, \sigma)$. Slightly abusing notation view $\sigma$ as a permutation of the numbers $\{1, \ldots, i\}$. Starting with $X_{i}$ and performing first the permutation $\sigma$ and then applying the strict descending operation associated to $\bar{\varepsilon}^{\prime}=(i, \sigma(k), t)$, yields the tuple $X_{i} \sigma \varepsilon^{\prime}$ whose underlying set is the same as that of the tuple $X_{i} \varepsilon$. Therefore there is some permutation $\sigma^{\prime}$ such that the tuples $X_{i} \sigma \varepsilon^{\prime}$ and $X_{i} \varepsilon \sigma^{\prime}$ coincide. And this implies that we have a relator $\bar{\sigma} \cdot \bar{\varepsilon}^{\prime}=\bar{\varepsilon} \cdot \bar{\sigma}^{\prime}$ or

$$
(i, \sigma)(i, \sigma(k), t)=(i, k, t)\left(i+n_{t}-1, \sigma^{\prime}\right) .
$$

\section{A finite generating set}

In this section, we show that the generating system $W_{s} \cup W_{p}$ can be reduced to a finite one. We begin with $W_{s}$. We will use the following two particular cases of relators of disjoint type. 
Case 1: Let $(i, k, t)$ be a triple such that $i-k>n_{l}-1$ for any colour $l$ where we include the case $l=t$. Assume moreover that the terminal point of the associated edge in $Z / G$, i.e., $\bar{X}_{i+n_{t}-1}$, is not a root of the tree $\mathcal{T}$. Recall that this edge consists of applying a descending operation of colour $t$, which increases the cardinality in $n_{t}-1$. Then there is some edge of $\mathcal{T}$ ending in $\bar{X}_{i+n_{t}-1}$. Let $s$ be the colour of this last edge which is represented as a triple by $\left(i+n_{t}-n_{s}, i+n_{t}-n_{s}, s\right)$ (recall that we constructed the tree $\mathcal{T}$ in such a way that the last element of each tuple is always being expanded). Now, as $i-k>n_{s}-1$ we deduce $k<i-n_{s}+1$. Thus there is a relator of disjoint type such as in (2) but with $i-n_{s}+1$ instead of $i$, $k$ instead of $k_{1}$ and $i-n_{s}+1$ instead of $k_{2}$. This relator is

$$
\left(i-n_{s}+1, k, t\right)\left(i+n_{t}-n_{s}, i+n_{t}-n_{s}, s\right)=\left(i-n_{s}+1, i-n_{s}+1, s\right)(i, k, t) .
$$

Since there is also a relator

$$
\left(i+n_{t}-n_{s}, i+n_{t}-n_{s}, s\right)=1,
$$

because it belongs to $\mathcal{T}$, we deduce

$$
(i, k, t)=\left(i-n_{s}+1, i-n_{s}+1, s\right)^{-1}\left(i-n_{s}+1, k, t\right) .
$$

This means that $(i, k, t)$ can be expressed in terms of triples with a smaller value of $i$. Case 2: Let $(i, k, t)$ be a triple such that $i \geq k \geq n_{t}+1$. Then $k-n_{t}+1>1$ and $i-n_{t}+1 \geq 2$. This means that there is a relator of disjoint type such as in (2) but with $i-n_{t}+1$ instead of $i, 1$ instead of $k_{1}$ and $k-n_{t}+1$ instead of $k_{2}$. This relator is

$$
\left(i-n_{t}+1,1, t\right)(i, k, t)=\left(i-n_{t}+1, k-n_{t}+1, t\right)(i, 1, t) .
$$

From this we deduce

$$
(i, k, t)=\left(i-n_{t}+1,1, t\right)^{-1}\left(i-n_{t}+1, k-n_{t}+1, t\right)(i, 1, t),
$$

meaning that $(i, k, t)$ can be expressed in terms of triples with either a smaller value of $i$ or with $k=1$.

Observe now that arguing by induction on $i+k$, equations (4) and (5) imply that any element in $W_{s}$ lies in the finite subgroup generated by the finite subset

$\left\{g \in W_{s} \mid\right.$ the associated triple fails to fulfil

both the conditions in Case 1 and in Case 2\}.

Example 5.1. Let us consider the group $V$, i.e., where we have one colour $t$ and $n_{t}=2$. For now let us only concentrate on the strict generators $W_{s}$. Note that an element $(i, i, t)$ is the identity. Looking at the representation by tree-pair diagrams, and the choice of $X_{i}$ in Example 4.3, we see that we expand the rightmost leaf of the rightmost tree $X_{i}$, hence we obtain $X_{i+1}$ and the group element is represented by $\left(X_{i+1}, X_{i+1}\right)$, which is the identity. Now consider elements $(i, k, t)$, where 
$k<i-1$. Again, using the rightmost-tree, we see that after deleting unnecessary carets on the right, we get

$$
(i, k, t)=(k+1, k, t),
$$

which is exactly the relator (4). For example, consider $(3,1, t)$. Then the corresponding tree-pair diagram is
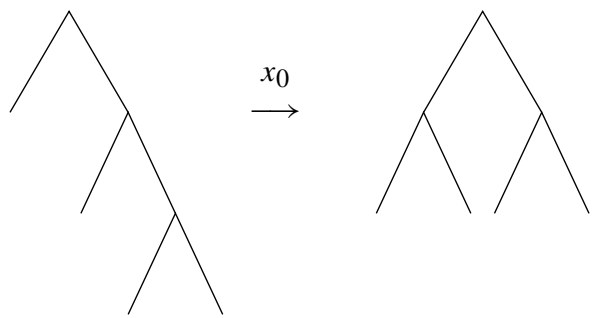

$X_{4}$

$$
X_{4}^{\prime}=X_{3} \varepsilon
$$

In particular, after deleting the rightmost caret in each tree, this is exactly the element $x_{0}$, see the picture after Remark 2.4.

Writing

$$
x_{i-2}=(i, i-1, t),
$$

we recover the well-known infinite generating set $\left\{x_{k} \mid k \geq 0\right\}$ for $F<V$. Furthermore, this enables us to simplify the relator (2) above. We have

$$
\left(i, k_{1}, t\right)\left(i+1, k_{2}+1, t\right)=\left(i, k_{2}, t\right)\left(i+1, k_{1}, t\right) .
$$

Using that $(i, k, t)=(k+1, k, t)$ for $k<i-1$, we get the well-known relator

$$
x_{k}^{-1} x_{l} x_{k}=x_{l+1}
$$

for any $k$ and $l$. Moreover, observe that strict generators and disjoint relators give us the well-known infinite presentation of Thompson's group $F$; see [Cannon et al. 1996].

Now we want to reduce $W_{p}$ in a similar way. The most natural way to do that is using relators of type $R_{P D}$, i.e., those mixing permutations and strict generators. To be able to argue by induction as before, we need to show that if $i$ is big enough, any element of the form $\left(X_{i}, \sigma\left(X_{i}\right)\right)$, where $\sigma$ is a permutation, can be expressed in terms of permutations with a smaller $i$ and possibly strict generators. As the group of permutations of the tuple $X_{i}$ is generated by transpositions, we may assume that $\sigma$ itself is a transposition. Now, assume that $i \geq 3 n_{t}$ for $t$ a colour with smallest possible arity $n_{t}$. As $\sigma$ only moves two elements, we may find $n_{t}$ consecutive elements in $X_{i}$ which are untouched by $\sigma$. Let $k$ be such that the $k$-th element in $X_{i}$ is the first one of those $n_{t}$ consecutive elements, and consider the strict generator associated to the triple $\left(i-n_{t}+1, k, t\right)$. Let $\sigma^{\prime}$ be the transposition of $X_{i-n_{t}+1}$ 
that moves precisely the elements that are also moved by $\sigma$. Then the associated relator (3) with $i-n_{t}+1$ instead of $i$, and $\sigma$ and $\sigma^{\prime}$ interchanged is

$$
\left(i-n_{t}+1, \sigma^{\prime}\right)\left(i-n_{t}+1, k, t\right)=\left(i-n_{t}+1, k, t\right)(i, \sigma) .
$$

Thus

$$
(i, \sigma)=\left(i-n_{t}+1, k, t\right)^{-1}\left(i-n_{t}+1, \sigma^{\prime}\right)\left(i-n_{t}+1, k, t\right)
$$

as we wanted to show.

This discussion can be summarised as follows:

Theorem 5.2. Assume that $U_{r}(\Sigma)$ is valid and bounded. Then $V_{r}(\Sigma)$ is generated by the finite set consisting of elements of the following three types:

(1) Strict generators associated to triples $(i, k, t)$ with $i \leq n_{t}+1$ and $i-k \leq n_{s}$ for any colour s.

(2) Strict generators associated to triples $(i, k, t)$ such that $\bar{X}_{i+n_{t}-1}$ is a root of the tree $\mathcal{T}$.

(3) Permutations associated to pairs $(i, \sigma)$ such that $i<3 n_{t}$ for some colour $t$.

Example 5.3. Consider $G=V$. In Example 5.1, we have already recovered the infinite presentation for $F<V$. In the tree of Example 4.3, the triples have a single root $X_{1}$ so we do not have to consider generators as in item (2) of Theorem 5.2. As before, let $i \geq 2$ and denote by $x_{i-2}$ the group element associated to the triple $(i, i-1, t)$. Then from Theorem 5.2 one deduces the well-known fact that the elements $x_{i}, i \geq 1$, together with the permutations generate the group and that $x_{0}$ and $x_{1}$ plus permutations are enough.

Remark 5.4. Similar generating systems can be obtained without using the space $Z$ by proceeding as Burillo and Cleary did for the Brin-Thompson groups $s V$ [Burillo and Cleary 2010, Theorem 2.1]. Instead of our first step (Section 4A), fix a set of tuples, one for each possible cardinality, which are to be the "source tree" of our tree pairs, and as "target tree" we allow anything that is obtained from one of these tuples by descending operations and permutations only. If $g \in G$ is an arbitrary element, it follows from the fact that any two bases have a common descendant that $g=\left(Y_{1}, Y_{2}\right)$ where $Y_{1}$ and $Y_{2}$ are obtained in that way. Then, choose $X_{i}$ in our previously fixed set of tuples (what used to be the set of nodes in $\mathcal{T}$ ) of the same cardinality as $Y_{1}$ and $Y_{2}$, and observe that $g=g_{2} g_{1}^{-1}$ with $g_{1}=\left(X_{i}, Y_{1}\right)$ and $g_{2}=\left(X_{i}, Y_{2}\right)$. These are precisely the type of elements we wanted to verify to be the generators of the group.

The choice of that fixed set of tuples can be the same as in Section 4B, but now we no longer need to construct the actual tree $\mathcal{T}$, we only need the nodes. For example, we can proceed as follows: as done before, fix a tuple $X_{r}$ with $r$ elements 
and choose integers $m_{1}, \ldots, m_{s}$ with

$$
d=\sum_{t=1}^{s} m_{t}\left(n_{t}-1\right) .
$$

There is a sequence of operations (first descending, then ascending) that we can perform on the last element of $X_{r}$ to get a new tuple with exactly $r+d$ elements that we denote $X_{r} \tau$. We may repeat the process to get a new tuple $X_{r} \tau^{2}$ and so on. We set $X_{r} \tau^{0}:=X_{r}$, let $X_{i+r d}:=X_{r} \tau^{i}$ for $i \geq 0$ and take the obtained family as our prefixed set of "sources".

As seen above, our first set of generators is then

$$
\left\{\left(X_{j}, X_{i} \varepsilon\right) \mid \varepsilon \text { is a sequence of descending operations }\right\} .
$$

Using Section 4D this can be further reduced to

$$
\left\{\left(X_{j}, X_{i} \varepsilon\right) \mid \varepsilon \text { is a single strict descending operation or permutation }\right\} .
$$

Again, there is no serious need of the space $Z$ to see that this reduction is possible. One can just check that composition of these elements corresponds to composition of the associated descending operations, in a way similar to that of [Burillo and Cleary 2010]. The same happens with the reduction performed in Section 4E: basically, we used $Z$ only to have some identities available that allowed us to eliminate some elements from our generating system, but all those identities can be easily checked by hand and one gets the same finite set in the end.

\section{Finite presentations}

In this section, we still assume that $U_{r}(\Sigma)$ is valid and bounded and we add the extra hypothesis that it is also complete to exhibit a procedure that gives a finite presentation. To do that, we just replace $Z$ by a truncated version $Z^{n}$ and we use the results of Section 4 to obtain an explicit finite presentation.

Definition 6.1. Using the notation of Definition 2.1, suppose that for all $i \neq i^{\prime}$, $i, i^{\prime} \in S$ we have that $\Sigma_{2}^{i, i^{\prime}} \neq \varnothing$ and that $f(j)=i^{\prime}$ for all $j=1, \ldots, n_{i}$ and $f^{\prime}\left(j^{\prime}\right)=i$ for all $j^{\prime}=1, \ldots, n_{i^{\prime}}$. Then we say that $U_{r}(\Sigma)$ is complete.

Considering the Morse function $t(A)=|A|$ in $\mathcal{S}_{r}(\Sigma)$ we can filter the complex with respect to $t$, and define the truncated Stein complex

$$
\mathcal{S}_{r}(\Sigma)^{n}:=\text { full subcomplex supported on }\left\{A \in \mathcal{S}_{r}(\Sigma) \mid t(A) \leq n\right\} .
$$

In particular this is just the simplicial complex $\mathcal{S}_{r}(\Sigma)^{n}$ obtained by considering bases of cardinality bounded by $n$ only. Note that in [Martínez-Pérez et al. 2016, Theorem 3.1] this complex was used to show that under the conditions above $V_{r}(\Sigma)$ 
is of type $F_{\infty}$. The purpose of this section is to give a recipe for constructing explicit presentations.

Obviously, we can do the same with the complex $Z$ and consider its truncated version $Z^{n}$ where the tuples have at most $n$ elements. The map $u$ restricts to these truncated versions and the same argument as in Section 3B shows that there is a homotopy equivalence

$$
u: Z^{n} \rightarrow\left|\mathcal{S}_{r}(\Sigma)^{n}\right| .
$$

By [Fluch et al. 2013, Corollary 3.9] for the special case of $s V$ and [Martínez-Pérez et al. 2016, Section 3] for the general case, assuming that $U_{r}(\Sigma)$ is valid, bounded and complete, there is some positive integer $n_{0}$ depending on $\Sigma$, such that for any $n \geq n_{0}$ and any basis $B \in \mathcal{S}_{r}(\Sigma)$ with cardinality $|B|=n+1$ the descending link of $B$ in the Stein complex $\mathcal{S}_{r}(\Sigma)$ is simply connected. Using Morse theory ([Bestvina and Brady 1997, Corollary 2.6]) we deduce that for $n \geq n_{0}$ the inclusion

$$
\mathcal{S}_{r}(\Sigma)^{n} \subseteq \mathcal{S}_{r}(\Sigma)^{n+1}
$$

induces an isomorphism in $\pi_{1}$ and $\pi_{0}$. As the space $\mathcal{S}_{r}(\Sigma)$ is contractible we have

$$
\begin{aligned}
& 1=\pi_{1}\left(\mathcal{S}_{r}(\Sigma)\right)=\lim \pi_{1}\left(\mathcal{S}_{r}(\Sigma)^{n}\right), \\
& 1=\pi_{0}\left(\mathcal{S}_{r}(\Sigma)\right)=\lim \pi_{0}\left(\mathcal{S}_{r}(\Sigma)^{n}\right),
\end{aligned}
$$

and $1=\pi_{1}\left(\mathcal{S}_{r}(\Sigma)^{n}\right)=\pi_{0}\left(\mathcal{S}_{r}(\Sigma)^{n}\right)$ for $n \geq n_{0}$. From this we deduce that $\mathcal{S}_{r}(\Sigma)^{n}$ is path connected and simply connected for $n \geq n_{0}$. This, together with the fact that $u$ is a homotopy equivalence, implies that the same holds true for $Z^{n}$. Finally, observe that $Z^{n}$ being path connected implies that the same is true for $Z^{n} / G$. Therefore we can use $Z^{n}$ instead of $Z$ in Theorem 4.1 and as $Z^{n} / G$ is finite we get a finite presentation. Hence we have the following theorem.

Theorem 6.2. Let $U_{r}(\Sigma)$ be a valid, bounded and complete Cantor algebra, and let $n \geq 1$ be such that $Z^{n}$ is simply connected. Then there is a finite presentation of $V_{r}(\Sigma)$ involving only strict generators $(i, k, t)$ with $i+n_{t}-1 \leq n$, permutations $(i, \sigma)$ with $i \leq n$, and relators involving these generators only, and which is obtained by truncating the presentation

$$
\left\langle W_{s} \cup W_{p} \mid R_{D} \cup R_{\Sigma} \cup R_{P D} \cup R_{P}\right\rangle
$$

given in (1).

The main difference with the reduction process of Section 5 is that we are now also reducing the set of relators. Moreover, the "truncated" set of generators in the finite presentation obtained this way can be further reduced using the same arguments as in Section 5. 
Example 6.3. For $G=V$, in [Fluch et al. 2013, Corollary 3.9] there is an explicit condition on $n$ that implies that $Z^{n}$ is simply connected: we need

$$
1 \leq\left\lfloor\frac{n-1}{3}\right\rfloor-1
$$

thus we can take $n=7$. This means that the set of strict generators in Example 5.1 can be reduced to $x_{0}, \ldots, x_{4}$ and the relators of disjoint type can be reduced to

$$
x_{k}^{-1} x_{l} x_{k}=x_{l+1},
$$

where $(k, l, l+1)$ is one of the following tuples: $(0,1,2),(0,2,3),(0,3,4)$, $(1,2,3),(1,3,4),(2,3,4)$. At this point it is not difficult to write down a finite presentation of $V$. Note also that in Example 5.3 we had already reduced to two strict generators $x_{0}$ and $x_{1}$.

Recently, Bleak and Quick [2017] found a short finite presentation for $V$ with two generators and nine relations using different methods.

Using our methods we get a finite presentation of Thompson's group $F$, and by using Tietze moves this presentation can be transformed to the well-known

$$
\left\langle x_{0}, x_{1} \mid x_{0}^{-3} x_{1} x_{0}^{3}=x_{1}^{-1} x_{0}^{-2} x_{1} x_{0}^{2} x_{1}, x_{0}^{-2} x_{1} x_{0}^{2}=x_{1}^{-1} x_{0}^{-1} x_{1} x_{0} x_{1}\right\rangle
$$

Example 6.4. For $G=s V$ we can also use [Fluch et al. 2013, Corollary 3.9] to compute the value of $n$ making $Z^{n}$ simply connected: we need

$$
1 \leq\left\lfloor\frac{n-1}{2^{s}}\right\rfloor-1
$$

thus we can take $n=1+2^{s+1}$. Recall that when choosing the maximal tree in $Z / G$ we chose expansion by one colour only (see Example 4.3). Let that colour be denoted by 1. For the same reason as in Example 5.1 we now have that elements of the form $(i, i, 1)$ are the identity, and that for any colour $t$ and any $k<i-1$, we have $(i, k, t)=(k+1, k, t)$.

This now gives an infinite $W_{s}$, which for $G=2 V$ can be listed as

$$
(i+1, i, 1), \quad(i+1, i, 2), \quad \text { and } \quad(k, k, 2),
$$

which corresponds to the infinite order generators $A_{i-1}, B_{i-1}$ and $C_{i}$ of Brin's infinite generating set of $2 V$; see [Brin 2004] or [Burillo and Cleary 2010]. Now by Theorem 5.2(1), this can be reduced to a finite generating set with seven strict generators; those where $i \leq 2$ and $k \leq 3$, as well as a finite number of permutation generators. Using Theorem 6.2 without any further reductions, we get a finite presentation where $i \leq 7$ and $k \leq 8$. 


\section{Finite presentation for centralisers of finite subgroups}

The proof of [Martínez-Pérez et al. 2016, Theorem 4.9] can be used to show that whenever the group $V_{k}(\Sigma)$ is finitely presented for any $k$, then so is $C_{V_{r}(\Sigma)}(Q)$ for any finite $Q \leq V_{r}(\Sigma)$, but the proof there does not yield an explicit finite presentation. In this section we are going to construct a finite presentation of $C_{V_{r}(\Sigma)}(Q)$. To do that, we proceed as follows. Note first that, by [Martínez-Pérez et al. 2016, Theorem 4.2], the group $C_{V_{r}(\Sigma)}(Q)$ is a direct product of groups of the form

$$
\underset{\lim _{(}}{\longrightarrow}\left(U_{r^{\prime}}(\Sigma), L\right) \rtimes V_{r^{\prime}}(\Sigma) \text {. }
$$

We now summarise the notation developed in [Martínez-Pérez et al. 2016]. The semidirect product above is associated to a fixed transitive permutation representation $\varphi: Q \rightarrow S_{m}$ of the finite group $Q$, where $S_{m}$ is the symmetric group of degree $m$, the orbit length. Then $L$ is the centraliser of the image $\varphi(Q)$ in $S_{m}$ and thus is a finite group. The number $r^{\prime}$ depends on $\varphi$ (see [Martínez-Pérez et al. 2016, Theorem 4.2]), but in order to simplify notation we will just set $r^{\prime}=r$. The set of bases in $U_{r}(\Sigma)$ together with the expansion maps can be viewed as a directed graph and we let $\left(U_{r}(\Sigma), L\right)$ be the following diagram of groups associated to this graph: To each basis $A$ we associate $\operatorname{Maps}(A, L)$, the group with elements the maps from $A$ to $L$ where the group operation is induced by multiplication in $L$. Each simple expansion $A \leq B$ corresponds to the diagonal map $\delta: \operatorname{Maps}(A, L) \rightarrow \operatorname{Maps}(B, L)$ with $\delta(f)\left(a \alpha_{i}^{j}\right)=f(a)$, where $a \in A$ is the expanded element. Then we consider the direct limit $\lim _{(}\left(U_{r}(\Sigma), L\right)$ whose elements are determined by some basis $A$ and a map $A \rightarrow L$. Observe that we may always assume that the basis $A$ satisfies $X_{r} \leq A$.

We begin by studying presentations for $\lim _{(}\left(U_{r}(\Sigma), L\right)$. We will obtain an infinite presentation (see Lemma 7.1 below) and then we will use the semidirect product action of $V_{r}(\Sigma)$ on this presentation together with the so-called Burnside procedure described in the Appendix to get a (finite) presentation of the group $\underset{\lim }{\longrightarrow}\left(U_{r}(\Sigma), L\right) \rtimes V_{r}(\Sigma)$.

We begin by constructing a generating system for the group $\lim _{\longrightarrow}\left(U_{r}(\Sigma), L\right)$. Take $x \in L$ and $A$ a basis with $X_{r} \leq A$. Take some subset $A_{1} \subseteq A$ and let $\chi_{A_{1}, x} \in$ $\underline{\lim _{(}}\left(U_{r}(\Sigma), L\right)$ be the element that maps every $a \in A_{1}$ to $x$ and every $a \in A \backslash A_{1}$ to the identity $1 \in L$. It is easy to see that the set of all the elements of this form generates our group, but observe that there might be a uniqueness issue because if we had another basis $C$ with $A \leq C$ and $C_{1}$ were the subset of those elements in $C$ coming from elements in $A_{1}$, then $\chi_{A_{1}, x}$ would equal $\chi_{C_{1}, x}$. To avoid this problem we set $\omega\left(A_{1}\right):=\left\{b\right.$ is a descendant of elements in $X_{r} \mid a w=b w^{\prime}$

for some $a \in A_{1}$ and descending words $\left.w, w^{\prime}\right\}$

(this was denoted $A_{1}(\mathcal{L})$ in [Martínez-Pérez et al. 2016]) and

$$
\Omega:=\left\{\omega\left(A_{1}\right) \mid A_{1} \text { is a subset of some basis } A \geq X_{r}\right\} .
$$


At first sight, this set $\Omega$ seems different from the set $\Omega$ defined in [Martínez-Pérez et al. 2016], which was defined for arbitrary finite subsets of the set of all descendants of elements in $X_{r}$, but Lemma 4.5(i) in that paper shows that since we are assuming that our Cantor algebra is valid and bounded they are in fact equal.

We set $\chi_{\omega, x}:=\chi_{A_{1}, x}$, where $\omega=\omega\left(A_{1}\right)$. Observe that the proof of [MartínezPérez et al. 2016, Lemma 4.5(i)] also implies that $\omega\left(A_{1}\right)=\omega\left(C_{1}\right)$, provided that $A \leq C$ and $C_{1}$ is the subset of those elements in $C$ coming from elements in $A_{1}$ (or, in other words, $C_{1}=C \cap \omega\left(A_{1}\right)$ ). As a consequence one easily sees that for any $B_{1}$ subset of a basis $B$ with $X_{r} \leq B$,

$$
\chi_{A_{1}, x}=\chi_{B_{1}, x} \Longleftrightarrow \omega\left(A_{1}\right)=\omega\left(B_{1}\right),
$$

implying that $\chi_{\omega, x}$ is well defined.

We will need a bit more of the notation from [Martínez-Pérez et al. 2016]. Let $\omega \in \Omega$ and $A_{1} \subseteq A \geq X_{r}$ with $\omega=\omega\left(A_{1}\right)$. We set

$$
\|\omega\|= \begin{cases}t & \text { if }\left|A_{1}\right| \equiv t \bmod d \text { with } 0<t \leq d, \\ 0 & \text { if } \omega=\varnothing .\end{cases}
$$

This does not depend on $A_{1}$; see [Martínez-Pérez et al. 2016, Lemma 4.5(v)]. Now, let $\omega_{1}, \omega_{2} \in \Omega$ and $A_{1}, A_{2} \subseteq A \geq X_{r}$ with $\omega_{i}=\omega\left(A_{i}\right)$ for $i=1,2$. Observe that the fact that our Cantor algebra is bounded means that we can always find such $A_{1}$ and $A_{2}$. If $A_{1} \cap A_{2}=\varnothing$, we write $\omega_{1} \wedge \omega_{2}=\varnothing$. Again, this is well defined, by [Martínez-Pérez et al. 2016, Lemma 4.5(vi)].

Lemma 7.1. The following is a presentation of $\underline{\lim }\left(U_{r}(\Sigma), L\right)$ :

$$
\left\langle\left(\chi_{\omega, x}\right)_{\omega \in \Omega \backslash \varnothing, x \in L} \mid \mathcal{R}_{1}, \mathcal{R}_{2}, \mathcal{R}_{3}\right\rangle,
$$

where

$$
\begin{aligned}
& \mathcal{R}_{1}=\left\{\chi_{\omega, x y}^{-1} \chi_{\omega, x} \chi_{\omega, y} \mid \omega \in \Omega, x, y \in L\right\}, \\
& \mathcal{R}_{2}=\left\{\left[\chi_{\omega, x}, \chi_{\omega^{\prime}, y}\right] \mid \omega, \omega^{\prime} \in \Omega, \omega \wedge \omega^{\prime}=\varnothing\right\}, \\
& \mathcal{R}_{3}=\left\{\chi_{\omega, x}^{-1} \chi_{\omega_{1}, x} \chi_{\omega_{2}, x} \mid \omega, \omega_{1}, \omega_{2} \in \Omega, \omega=\omega_{1} \dot{\cup} \omega_{2}\right\},
\end{aligned}
$$

where $\omega_{1} \dot{\cup} \omega_{2}$ denotes the disjoint union. Moreover $V_{r}(\Sigma)$ acts by permutations with finitely many orbits on this presentation.

Proof. As observed above, any $\chi \in \underline{\lim }\left(U_{r}(\Sigma), L\right)$ is a product of elements of the form $\chi_{\omega, x}$ for a suitable $\omega \in \Omega$ and $x \in L$. Let $F$ denote the free group on the set $\left\{\tilde{\chi}_{\omega, x} \mid \omega \in \Omega \backslash \varnothing, x \in L\right\}$. There is an epimorphism

$$
F \stackrel{\tau}{\rightarrow} \underset{\lim }{\longrightarrow}\left(U_{r}(\Sigma), L\right)
$$

with $\tau\left(\tilde{\chi}_{\omega, x}\right)=\chi_{\omega, x}$. Let $G$ be the abstract group defined in the statement of the result for the generators $\tilde{\chi}_{\omega, x}$. It is immediate to verify that the epimorphism $\tau$ 
defined above induces an epimorphism from $G$ to $\underline{\lim }\left(U_{r}(\Sigma), L\right)$ which we still call $\tau$. This follows since all relations inside $G$ are easily verified to hold for the images $\tau\left(\tilde{\chi}_{\omega, x}\right)$. Assume that we have a word $\widetilde{w}=w\left(\tilde{\chi}_{\omega_{1}, x_{1}}, \ldots, \tilde{\chi}_{\omega_{k}, x_{k}}\right)$, for some $\omega_{1}, \ldots, \omega_{k} \in \Omega$ and $x_{1}, \ldots, x_{k} \in L$. Assume further that

$$
1=\tau(\widetilde{w})=\tau\left(w\left(\tilde{\chi}_{\omega_{1}, x_{1}}, \ldots, \tilde{\chi}_{\omega_{k}, x_{k}}\right)\right)=w\left(\tau\left(\tilde{\chi}_{\omega_{1}, x_{1}}\right), \ldots, \tau\left(\tilde{\chi}_{\omega_{k}, x_{k}}\right)\right) .
$$

Let $X_{r} \leq A$ be a basis with subsets $A_{i} \subseteq A$ such that $\omega_{i}=A_{i}(\mathcal{L})$ for $i=1, \ldots, k$. We now refine the set $\left\{A_{1}, \ldots, A_{k}\right\}$ to a set $\left\{A_{1}^{\prime}, \ldots, A_{k^{\prime}}^{\prime}\right\}$ of subsets of $A$ such that for all $i, j \leq k^{\prime}$ either $A_{i}^{\prime} \cap A_{j}^{\prime}=\varnothing$ or $A_{i}^{\prime}=A_{j}^{\prime}$. By suitably applying the relations in $\mathcal{R}_{3}$ to both the original word $w\left(\widetilde{\chi}_{\omega_{1}, x_{1}}, \ldots, \tilde{\chi}_{\omega_{k}, x_{k}}\right)$ and its image

$$
w:=\tau(\widetilde{w})=w\left(\chi_{\omega_{1}, x_{1}}, \ldots, \chi_{\omega_{k}, x_{k}}\right),
$$

we may rewrite each occurrence of $\chi_{\omega_{i}, x_{i}}$ and $\tilde{\chi}_{\omega_{i}, x_{i}}$ in terms of suitable new elements $\tau\left(\widetilde{\chi}_{\omega_{j}^{\prime}, y_{j}}\right)$ and $\chi_{\omega_{j}^{\prime}, y_{j}}$ for $1 \leq j \leq k^{\prime}$, so that either $\omega_{j}^{\prime} \wedge \omega_{i}^{\prime}=\varnothing$ or $\omega_{j}^{\prime}=\omega_{i}^{\prime}$.

Reordering them so that $\omega_{1}, \ldots, \omega_{u}$ for $1 \leq u \leq k^{\prime}$ are pairwise distinct and applying the relations in $\mathcal{R}_{2}$ and $\mathcal{R}_{1}$ to group together the suitable products of the $y_{j}$ 's we obtain new words

$$
\widetilde{w} \sim \widetilde{w}^{\prime}=\tilde{\chi}_{\omega_{1}^{\prime}, z_{1}} \cdots \tilde{\chi}_{\omega_{u}^{\prime}, z_{u}}, \quad w \sim w^{\prime}=\chi_{\omega_{1}^{\prime}, z_{1}} \cdots \chi_{\omega_{u}^{\prime}, z_{u}},
$$

where the $\omega_{i}^{\prime}$ 's are pairwise disjoint.

If $w^{\prime} \sim 1$, we must have $z_{i}=1$ for any $1 \leq i \leq u$, by applying the word $w^{\prime}$ to an $a \in A_{i}$ such that $A_{i}(\mathcal{L})=\omega_{i}^{\prime}$. From $\mathcal{R}_{1}$ it is immediate to see that $\tilde{\chi}_{\omega, 1}=1$ for any $\omega \in \Omega$ so we also have $\widetilde{w} \sim \widetilde{w}^{\prime} \sim 1$ and $G$ gives a presentation of $\lim _{\longrightarrow}\left(U_{r}(\Sigma), L\right)$.

By [Martínez-Pérez et al. 2016, Lemma 4.7], the group $V_{r}(\Sigma)$ acts by permutations on $\Omega$. Moreover, for any $g \in V_{r}(\Sigma)$, if $\omega, \omega^{\prime} \in \Omega$ are such that $\omega \wedge \omega^{\prime}=\varnothing$, then $g \omega \wedge g \omega^{\prime}=\varnothing$ and if $\omega=\omega_{1} \cup \omega_{2}$ for $\omega_{1}, \omega_{2} \in \Omega$, then $g \omega=g \omega_{1} \cup g \omega_{2}$. Therefore $V_{r}(\Sigma)$ acts by permutations on this presentation. To prove the last statement, it suffices to check the following:

Claim 1. The set of generators is $V_{r}(\Sigma)$-finite.

Claim 2. Each of the sets of relations $\mathcal{R}_{1}, \mathcal{R}_{2}, \mathcal{R}_{3}$ is $V_{r}(\Sigma)$-finite.

As the group $L$ is finite, both claims follow from slight variations of the proof of [Martínez-Pérez et al. 2016, Lemma 4.7]. For example, for Claim 2 for $\mathcal{R}_{2}$, it suffices to check that whenever we have $\omega, \omega^{\prime}, \widehat{\omega}, \widehat{\omega}^{\prime} \in \Omega$ with

$$
\omega \wedge \omega^{\prime}=\varnothing, \quad \widehat{\omega} \wedge \widehat{\omega}^{\prime}=\varnothing, \quad\|\omega\|=\|\widehat{\omega}\| \quad \text { and } \quad\left\|\omega^{\prime}\right\|=\left\|\widehat{\omega}^{\prime}\right\|,
$$

then there is some $g \in V_{r}(\Sigma)$ such that for any $x \in L$, we have $\chi_{\widehat{\omega}, x}=\chi_{\omega, x}^{g}$ and $\chi_{\widehat{\omega}^{\prime}, x}=\chi_{\omega^{\prime}, x}^{g}$. To get a suitable $g$, choose bases $X_{r} \leq A, \widehat{A}$ so that for $B, B^{\prime} \subseteq A$ 
and $\widehat{B}, \widehat{B}^{\prime} \subseteq \widehat{A}$, we have

$$
\begin{gathered}
\omega=\omega(B), \quad \omega^{\prime}=\omega\left(B^{\prime}\right), \quad \widehat{\omega}=\omega(\widehat{B}), \quad \widehat{\omega}^{\prime}=\omega\left(\widehat{B}^{\prime}\right), \\
|A|=|\widehat{A}|, \quad|B|=|\widehat{B}|, \quad\left|B^{\prime}\right|=\left|\widehat{B}^{\prime}\right| .
\end{gathered}
$$

The assumptions imply that $B \cap B^{\prime}=\varnothing=\widehat{B} \cap \widehat{B}^{\prime}$. So we may choose a $g \in V_{r}(\Sigma)$ with $g A=\widehat{A}, g B=\widehat{B}$ and $g B^{\prime}=\widehat{B}^{\prime}$.

In a completely analogous way one proves that for $\omega, \omega_{1}, \omega_{2}, \widehat{\omega}, \widehat{\omega}_{1}, \widehat{\omega}_{2} \in \Omega$ with $\omega=\omega_{1} \cup \omega_{2}, \quad \widehat{\omega}=\widehat{\omega}_{1} \cup \widehat{\omega}_{2}, \quad\|\omega\|=\|\widehat{\omega}\|, \quad\left\|\omega_{1}\right\|=\left\|\widehat{\omega}_{1}\right\|, \quad\left\|\omega_{2}\right\|=\left\|\widehat{\omega}_{2}\right\|$, there is some $g \in V_{r}(\Sigma)$ such that for any $x \in L$,

$$
\chi_{\widehat{\omega}, x}=\chi_{\omega, x}^{g}, \quad \chi_{\widehat{\omega}_{1}, x}=\chi_{\omega_{1}, x}^{g} \quad \text { and } \quad \chi_{\widehat{\omega}_{2}, x}=\chi_{\omega_{2}, x}^{g} .
$$

Proposition 7.2. Assume that the group $V_{r}(\Sigma)$ is finitely presented. Let $Q \leq V_{r}(\Sigma)$ be a finite subgroup. Given a finite presentation of $V_{r}(\Sigma)$, Lemma 7.1 together with Theorem A.3 yield an explicit finite presentation of $C_{V_{r}(\Sigma)}(Q)$.

Proof. By [Martínez-Pérez et al. 2016, Theorem 4.2], it suffices to construct an explicit finite presentation of a group of the form

$$
H=\lim _{(}\left(U_{r}(\Sigma), L\right) \rtimes V_{r}(\Sigma)
$$

when $L$ is an arbitrary finite group. Let $V_{r}(\Sigma)=\langle Z \mid T\rangle$ be a finite presentation of $V_{r}(\Sigma)$ and let

$$
\underset{\lim }{\longrightarrow}\left(U_{r}(\Sigma), L\right)=\langle Y \mid R\rangle
$$

be the presentation constructed in Lemma 7.1. We need to verify the hypotheses of Theorem A.3. In Lemma 7.1 we have already checked that the group $V_{r}(\Sigma)$ acts by permutations in this presentation and that there are only finitely many orbits under that action. We may therefore choose $Y_{0} \subseteq Y$ and $R_{0} \subseteq R$ to be finite sets of representatives of these orbits.

The argument in Section A1 thus implies that the group $H$ has the presentation

$$
\left\langle Y_{0}, Z \mid \widehat{R}_{0}, T,\left[\operatorname{Stab}_{V_{r}(\Sigma)}(y), y\right], y \in Y_{0}\right\rangle .
$$

We can give explicit descriptions of possible choices for the sets $Y_{0}, R_{0}$. Set $X_{r}=\left\{x_{1}, \ldots, x_{r}\right\}$ and let $\omega_{i}=\omega\left(\left\{x_{1}, \ldots, x_{i}\right\}\right)$ for $i=1, \ldots, r$. Then:

$$
Y_{0}=\left\{\chi_{\omega_{i}, z} \mid 1 \leq i \leq r, z \in L\right\} .
$$

To describe $R_{0}$, we are going to split it into three pairwise disjoint subsets $R_{0}=R_{0}^{1} \cup R_{0}^{2} \cup R_{0}^{3}$, according to the three subsets of relations $\mathcal{R}_{1}, \mathcal{R}_{2}$ and $\mathcal{R}_{3}$ of Lemma 7.1. The simplest one is $R_{0}^{1}$ :

$$
R_{0}^{1}=\left\{\chi_{\omega_{i}, z y}^{-1} \chi_{\omega_{i}, z} \chi_{\omega_{i}, y} \mid 1 \leq i \leq r, z \in L\right\}
$$


For $R_{0}^{2}, R_{0}^{3}$ it is more convenient to fix a basis $X_{r} \leq A$ with $|A| \geq 2 r$. Then we may choose

$$
\begin{aligned}
& R_{0}^{2}=\left\{\left[\chi_{\omega, z}, \chi_{\omega^{\prime}, z}\right] \mid z \in L, \omega=\omega\left(A_{1}\right), \omega^{\prime}=\omega\left(A_{1}^{\prime}\right), A_{1}, A_{1}^{\prime} \subseteq A, A_{1} \cap A_{1}^{\prime}=\varnothing\right\}, \\
& R_{0}^{3}=\left\{\chi_{\omega, z}^{-1} \chi_{\omega_{1}, z} \chi_{\omega_{2}, z} \mid z \in L, \omega_{1}=\omega\left(A_{1}\right), \omega_{2}=\omega\left(A_{2}\right), \omega=\omega_{1} \dot{\cup} \omega_{2}, A_{1}, A_{2} \subseteq A\right\} .
\end{aligned}
$$

Observe that these choices of $R_{0}^{2}$ and $R_{0}^{3}$ yield redundant presentations.

The previous presentation may not be finite because of all the relations needed to form $\left[\operatorname{Stab}_{V_{r}(\Sigma)}(y), y\right]$ where $y \in Y_{0}$. Notice that $g \in \operatorname{Stab}_{V_{r}(\Sigma)}(y)$ if and only if $g(\omega)=\omega$ where $y=\chi_{\omega, z}$ for some $z \in L$. By [Martínez-Pérez et al. 2016, Lemma 4.7] and the assumption on $V_{r}(\Sigma)$ we deduce that $\operatorname{Stab}_{V_{r}(\Sigma)}(y)$ is finitely generated by some generators $\mu_{1}, \ldots, \mu_{m}$.

Consider now the following $m$ relations, which are a subset of the stabiliser relations $\left[\operatorname{Stab}_{V_{r}(\Sigma)}(y), y\right]$ :

$$
\mu_{i} \chi_{\omega, z} \mu_{i}^{-1}=\chi_{\omega, z}, \quad i=1, \ldots, m .
$$

If $g \in \operatorname{Stab}_{V_{r}(\Sigma)}(y)$, then $g=w\left(\mu_{1}, \ldots, \mu_{m}\right)$ and the stabiliser relation $g \chi_{\omega, z} g^{-1}=$ $\chi_{\omega, z}$ is thus obtained by starting from relation (7) for some $i$ and then suitably conjugating this relation to build the word $w$.

Therefore, by Lemmas A.1 and A.2, the group $H$ has the finite presentation

$$
\left\langle Y_{0}, Z \mid \widehat{R}_{0}, T,\left[\mu_{i}, y\right], i=1, \ldots, m, y \in Y_{0}\right\rangle,
$$

where the elements $\mu_{1}, \ldots, \mu_{m}$ are expressed as words in the generators $Z$.

\section{Appendix: The Burnside procedure}

We shall now give an outline of the Burnside procedure used in the proof of Proposition 7.2. As mentioned in the Introduction, we do not claim any originality for this. For example, this procedure has been used, without proof, in [Guralnick et al. 2011]. We are not aware of any place where a proof is presented. Hence we include it here for completeness.

The goal is to find a small finite presentation of a group, in the cases where the following procedure can be applied. The idea is to look for a possibly infinite, but well-behaved, presentation of a group $G$ and a group $Q$ such that the action of $Q$ on the generators and relators of $G$ cuts them down to a very small number. At a later stage, the group $Q$ will be assumed to be a subgroup of $G$ and its action will return a new smaller presentation.

A1. Preliminary lemmas. The beginning of this procedure is general and we only require each of the groups $G$ and $Q$ to have a presentation, without any assumption on them. 
Let $G=\langle Y \mid R\rangle$ and $Q=\langle Z \mid T\rangle$ be groups. Let $Q$ act on $Y$ by permutations. Notice that $R \subseteq F(Y)$, where $F(Y)$ is the free group generated by $Y$, and observe that $Q$ also acts on $F(Y)$. We assume that $Q(R)=R$. Let $Y_{0}$ be a set of representatives for the $Q$-orbits in $Y$ and $R_{0}$ be a set of representatives for the $Q$-orbits in $R$. We observe that $R_{0} \subseteq F(Y)=\left\langle t\left(a_{0}\right) \mid a_{0} \in Y_{0}, t \in Q\right\rangle$, that is, we may express the elements of $R_{0}$ as products of the results of $Q$ acting on elements of $Y_{0}$. In the special case that $Q$ is a subgroup of $G$, we will be able to express elements in $R_{0}$ as products of conjugates of elements in $Y_{0}$ by elements in $Q$. Hence each element of $R_{0}$, seen as an element in $G$, can be written in more than one way and we fix an expression of the type $t_{1}\left(a_{1}\right) \cdots t_{k}\left(a_{k}\right)$ for such elements. We then define the set $\widehat{R}_{0} \subseteq\left\langle t a_{0} t^{-1} \mid a_{0} \in Y_{0}, t \in Q\right\rangle$ to be the set of fixed expressions for the elements of $R_{0}$, where we have replaced the action of $Q$ on $Y_{0}$ by the conjugation of elements. That is, if $t_{1}\left(a_{1}\right) \cdots t_{k}\left(a_{k}\right)$ is a fixed expression in $R_{0}$, the corresponding element in $\widehat{R}_{0}$ is $t_{1} a_{1} t_{1}^{-1} \cdots t_{k} a_{k} t_{k}^{-1}$. The set $\widehat{R}_{0}$ is thus a set of formal expressions which will be used later to express relations in the groups.

Lemma A.1. Following the notation previously defined, we have

$$
G \rtimes Q \cong\left\langle Y_{0}, Z \mid \widehat{R}_{0}, T,\left[\operatorname{Stab}_{Q}(y), y\right], y \in Y_{0}\right\rangle,
$$

where the semidirect product is given by the action of $Q$ on $G$ as follows: for all $g_{1}, g_{2} \in G$ and $t_{1}, t_{2} \in Q$, multiplication is given by

$$
\left(g_{1}, t_{1}\right)\left(g_{2}, t_{2}\right)=\left(g_{1} \cdot t_{1}\left(g_{2}\right), t_{1} t_{2}\right) .
$$

Proof. Let $H$ be the group presented by $\left\langle Y_{0}, Z\right| \widehat{R}_{0}, T$, $\left.\left[\operatorname{Stab}_{Q}(y), y\right], y \in Y_{0}\right\rangle$. Define the group homomorphism $\varphi: F\left(Y_{0} \cup Z\right) \rightarrow G \rtimes Q$ by sending $a_{0} \in Y_{0}$ to $\left(a_{0}, 1\right) \in G \rtimes Q$ and $c \in Z$ to $(1, c) \in G \rtimes Q$. By construction we see that

$$
\varphi(t) \varphi\left(a_{0}\right) \varphi(t)^{-1}=\left(t\left(a_{0}\right), 1\right)
$$

for any word $t \in Q$.

Claim 1. The map $\varphi$ induces a homomorphism $H \rightarrow G \rtimes Q$, which we still call $\varphi$.

Proof. If $d \in T$ is a relation in $H$, then $d=c_{1} \cdots c_{k}$, for some $c_{i} \in Z$, and $\varphi\left(c_{1}\right) \cdots \varphi\left(c_{k}\right)=(1,1)$. Let now $\widehat{b}_{0} \in \widehat{R}_{0}$ be a relation in $H$, then

$$
\widehat{b}_{0}=t_{1} a_{1} t_{1}^{-1} \cdots t_{k} a_{k} t_{k}^{-1},
$$

for some $a_{i} \in Y_{0}$ and $t_{i} \in Q$. Moreover, by applying (*), we get

$$
\prod_{i=1}^{k} \varphi\left(t_{i}\right) \varphi\left(a_{i}\right) \varphi\left(t_{i}\right)^{-1}=\left(\prod_{i=1}^{k} t_{i}\left(a_{i}\right), 1\right)=(1,1) .
$$


Finally let $a_{0} \in Y_{0}, t \in \operatorname{Stab}_{Q}\left(a_{0}\right)$. Thus we have, using $(*)$ again,

$$
\varphi(t) \varphi\left(a_{0}\right) \varphi(t)^{-1} \varphi\left(a_{0}\right)^{-1}=\left(t\left(a_{0}\right), 1\right)\left(a_{0}^{-1}, 1\right)=(1,1) .
$$

Now we just apply von Dyck's theorem.

Claim 2. The map $\varphi$ is surjective.

Proof. Any element $(1, t) \in\{1\} \times Q:=\{(1, s) \mid s \in Q\}$ can be written as $\left(1, c_{1} \cdots c_{k}\right)$ for suitable $c_{i} \in Z$ and so $\varphi(H)$ contains $\{1\} \times Q$. We observe that any element of $G \times\{1\}:=\{(h, 1) \mid h \in G\}$ can be written as $\left(t_{1}\left(a_{1}\right) \cdots t_{k}\left(a_{k}\right), 1\right)$ for suitable $a_{i} \in Y_{0}$ and $t_{i} \in Q$. By arguing as in Claim 1 we have $(g, 1)=\varphi\left(\prod_{i=1}^{k} t_{i} a_{i} t_{i}^{-1}\right)$. Thus, $\varphi(H) \geq\langle G \times\{1\},\{1\} \times Q\rangle=G \rtimes Q$.

Claim 3. The map $\varphi$ is injective.

Proof. Any element of $Y$ can be written as $t\left(a_{0}\right)$, for some $a_{0} \in Y_{0}$ and $t \in Q$. Define $\bar{Y}^{*}=\left\{t a_{0} t^{-1} \mid a_{0} \in Y_{0}, t \in Q\right\}$ to be the set of symbols of $Y$ where we have replaced the action of $Q$ with the conjugation of elements. We notice that, if $t\left(a_{0}\right)=s\left(a_{0}\right)$, then $t^{-1} s \in \operatorname{Stab}_{Q}\left(a_{0}\right)$ and we thus define an equivalence relation on $\bar{Y}^{*}$ by writing $t a_{0} t^{-1} \sim s a_{0} s^{-1}$ if and only if $t^{-1} s \in \operatorname{Stab}_{Q}\left(a_{0}\right)$. We define $\bar{Y}:=\bar{Y}^{*} / \sim$ to be the collection of equivalence classes.

If $a \in Y$ and $a=t\left(a_{0}\right)$, for some $a_{0} \in Y_{0}$ and $t \in Q$, we define an element $\bar{a}$ of $\bar{Y}$ by setting $\bar{a}=\left\{s a_{0} s^{-1} \mid t^{-1} s \in \operatorname{Stab}_{Q}\left(a_{0}\right)\right\}$. With this notation, we observe that $Q$ acts on $\bar{Y}$ through

$$
(s, \bar{a}) \rightarrow s \cdot \bar{a}:=\overline{s t a_{0} t^{-1} s^{-1}},
$$

for some $a_{0} \in Y_{0}, t \in Q$ such that $\bar{a}=\overline{t a_{0} t^{-1}}$. Also, notice that the map $\psi: Y \rightarrow \bar{Y}$ sending $a \mapsto \bar{a}$ is a $Q$-equivariant bijection, that is $\psi(s a)=s \psi(a)=s \cdot \bar{a}$ for all $s \in Q$. Hence the action of $Q$ on $Y$ is equivalent to the action of $Q$ on $\bar{Y}$. For each element $\bar{a} \in \bar{Y}$ we can fix a representative $t a_{0} t^{-1} \in F\left(Y_{0} \cup Z\right)$ and we call the set of representatives $\widehat{Y}$. By construction, every element $\widehat{b}_{0} \in \widehat{R}_{0}$ can be uniquely written as $\widehat{b}_{0}=t_{1} a_{1} t_{1}^{-1} \cdots t_{k} a_{k} t_{k}^{-1}$, so we define $\bar{R}_{0} \subseteq F(\bar{Y})$ be the set of elements

$$
\overline{t_{1} a_{1} t_{1}^{-1}} \cdots \overline{t_{k} a_{k} t_{k}^{-1}} \text {. }
$$

We then let $\bar{R} \subseteq F(\bar{Y})$ be the set of all elements $\overline{t t_{1} a_{1} t_{1}^{-1} t^{-1}} \cdots \overline{t t_{k} a_{k} t_{k}^{-1} t^{-1}}$, for any $t \in Q$.

With these definitions, it makes sense to say that the normal closure $F(\bar{R})^{F(\bar{Y})}$ inside $F(\bar{Y})$ is isomorphic to $F(R)^{F(Y)}$ inside $F(Y)$. Also notice that

$$
F(\bar{Y}) \cong F\left(\bar{Y}^{*} / \sim\right)=\left\langle\bar{Y}^{*} \mid R \sim\right\rangle,
$$

where $R \sim$ is the set of all relations of the type $t a_{0} t^{-1} \sim s a_{0} s^{-1}$ if and only if $t^{-1} s \in \operatorname{Stab}_{Q}\left(a_{0}\right)$. 
Let $w \in H$ be such that $\varphi(w)=(1,1)$. Let $w=c_{1} a_{1} c_{2} a_{2} \cdots a_{k} c_{k+1}$ for $a_{i} \in Y_{0}$ and $c_{i} \in\langle Z\rangle$ and we rewrite $w$ as

$$
w=\left(c_{1} a_{1} c_{1}^{-1}\right)\left(c_{1} c_{2} a_{2} c_{2}^{-1} c_{1}^{-1}\right) \cdots\left(c_{1} c_{2} \cdots c_{k} a_{k} c_{k}^{-1} \cdots c_{1}^{-1}\right) c_{1} c_{2} \cdots c_{k} c_{k+1} .
$$

Define $t_{i}=c_{1} \cdots \underline{c_{i}}$. Then, up to replacing $t_{i}$ with another suitable $t_{i}^{\prime} \in Q$, we can assume that $\overline{t_{i} a_{i} t_{i}^{-1}} \in \widehat{Y}$. Hence we can write $w=\left(t_{1} a_{1} t_{1}^{-1} \cdots t_{k} a_{k} t_{k}^{-1}\right) t_{k+1}$ and, applying $\varphi$ to the rewriting of $w$ we get $(1,1)=\left(t_{1}\left(a_{1}\right) \cdots t_{k}\left(a_{k}\right), t_{k+1}\right)$.

Since $t_{k+1}=1$ inside $Q$, we can use the relations of $Q$ to rewrite $t_{k+1}=1$ inside $H$. Similarly, since $t_{1}\left(a_{1}\right) \cdots t_{k}\left(a_{k}\right)=1$ inside $G$ and since the normal closure $F(\bar{R})^{F(\bar{Y})}$ inside $F(\bar{Y})$ is isomorphic to $F(R)^{F(Y)}$ inside $F(Y)$, we can use the relations of $G$ to rewrite $t_{1} a_{1} t_{1}^{-1} \cdots t_{k} a_{k} t_{k}^{-1}=1$ inside $H$. Therefore $w=1$ in $H$ and $\operatorname{so} \varphi$ is injective.

The map $\varphi$ is thus a group isomorphism and the proof of Lemma A.1 is complete.

The following result does not depend on the presentations of the relevant groups and relies only on the definition of semidirect product.

Lemma A.2. Let $G$ be a group and $Q \leq G$. Let $G \rtimes Q$ be the semidirect product constructed using the action of $Q$ on $G$ by conjugation inside $G$. Then

$$
G \rtimes Q \cong G \times Q .
$$

Proof. Let $H:=G \rtimes Q$ with product given by $(a, x)(b, y)=\left(a x b x^{-1}, x y\right)$. It is clear that $\widetilde{Q}=\left\{\left(t^{-1}, t\right) \mid t \in Q\right\}$ is a subgroup of $H$ and $\widetilde{Q} \cong Q$. Since

$$
(a, x)=(a x, 1)\left(x^{-1}, x\right),
$$

$H$ is generated by $G \times\{1\}$ and $\widetilde{Q}$. It is straightforward to verify that $Q$ is normal and so, since $G \times\{1\}$ is normal as well, we get $G \rtimes Q \cong(G \times\{1\}) \times \widetilde{Q} \cong G \times Q$.

A2. The Burnside procedure. We are now ready to explain the Burnside procedure. We make two additional assumptions with respect to those in Section A1. We assume

(i) the presentation $Q=\langle Z \mid T\rangle$ is finite,

(ii) the number of $Q$-orbits in $Y$ is finite (and possibly very small, in practical applications),

(iii) the number of $Q$-orbits in $R$ is finite (and also possibly very small),

(iv) the stabilisers $\operatorname{Stab}_{Q}(y)$ are finitely generated, for $y \in Y_{0}$.

Let $G$ and $Q$ be as defined in Lemma A.1, $Q \leq G$ and let $Q$ act by conjugation on $G$, then Lemmas A.1 and A.2 imply that

$$
\left.G \times Q \cong\left\langle Y_{0}, Z\right| \widehat{R}_{0}, T,\left[\operatorname{Stab}_{Q}(y), y\right] \text { for } y \in Y_{0}\right\rangle .
$$


We rewrite $Z$ in terms of $Y_{0}$ and then mod out $Q$. We also use the finite generation of $\operatorname{Stab}_{Q}(y)$ to rewrite the stabiliser relations as conjugations. Therefore we obtain the following theorem:

Theorem A.3 (Burnside procedure). Let $G, Q$ be the groups defined in Lemma A.1. Assume that

(i) $Q \leq G$ and $Q$ acts by conjugation on $G$,

(ii) $Q=\langle Z \mid T\rangle$ is finitely presented,

(iii) the number of $Q$-orbits in $Y$ is finite,

(iv) the number of $Q$-orbits in $R$ is finite,

(v) the stabilisers $\operatorname{Stab}_{Q}(y)$ are finitely generated, for $y \in Y_{0}$.

Then there exists a finite presentation of $G$ of the type

$$
\begin{aligned}
G=\left\langle Y_{0}, Z\right| R_{0}, T, c y c c^{-1}=y, \text { for } y \in Y_{0}, \\
\text { a generator } \left.c \text { of } \operatorname{Stab}_{Q}(y), \text { finitely many extra relations }\right\rangle,
\end{aligned}
$$

where the extra relations are obtained in the following way: there is a relation for every element $c \in Z$ and it has the form

$$
c=\text { word in conjugates of elements of } Y_{0} \text { by elements of } Z \text {. }
$$

A3. An application. The following example is taken from [Guralnick et al. 2011]. Recall the following presentation for the alternating group

$$
\operatorname{Alt}(n+2)=\left\langle x_{1}, \ldots, x_{p} \mid\left(x_{i}\right)^{3},\left(x_{i} x_{j}\right)^{2}, i \neq j\right\rangle,
$$

where $x_{i}$ can be realised as the 3-cycle $(i n+1 n+2)$. Hence

$$
\operatorname{Alt}(7)=\left\langle x_{1}, x_{2}, x_{3}, x_{4}, x_{5} \mid\left(x_{i}\right)^{3},\left(x_{i} x_{j}\right)^{2}, i \neq j\right\rangle:=G .
$$

On the other hand, it can be shown that

$$
\operatorname{Alt}(5)=\left\langle a, b \mid a^{5}, b^{2},(a b)^{3}\right\rangle:=Q,
$$

where $a$ can be realised as (1 2345$)$ and $b=(23)(45)$. Let $z:=x_{1}=\left(\begin{array}{ll}1 & 67\end{array}\right)$ and observe that $x_{i}=z^{a^{i-1}}$, for $i=1, \ldots, 5$. Now we check that

$$
\begin{aligned}
& Y=\left\{x_{1}, \ldots, x_{5}\right\}, \quad Y_{0}=\{z\}, \quad R=\left\{\left(x_{i}\right)^{3},\left(x_{i} x_{j}\right)^{2}, i \neq j\right\}, \\
& R_{0}=\left\{z^{3},\left(z z^{a}\right)^{2}\right\}, \quad Z=\{a, b\}, \quad T=\left\{a^{5}, b^{2},(a b)^{3}\right\}
\end{aligned}
$$

satisfy the conditions of Theorem A.3. Noting that $\left\{\left[\operatorname{Stab}_{Q}(y), y\right]\right.$ for $\left.y \in Y_{0}\right\}=$ $\left\{[z, b],\left[z,(b a)^{a}\right]\right\}$, we have

$$
G \times Q=\left\langle a, b, z \mid a^{5}, b^{2},(a b)^{3}, z^{3},\left(z z^{a}\right)^{2},[z, b],\left[z,(b a)^{a}\right]\right\rangle .
$$


We can write $a=w_{1}\left(x_{1}, \ldots, x_{5}\right)$ and $b=w_{2}\left(x_{1}, \ldots, x_{5}\right)$, for suitable words $w_{1}, w_{2} \in F\left(x_{1}, \ldots, x_{5}\right)$ and then Theorem A.3 yields the following finite presentation for $\operatorname{Alt}(7)$ :

$$
\begin{array}{r}
\operatorname{Alt}(7)=\langle a, b, z| R_{0}, T,[z, b],\left[z,(b a)^{a}\right], \\
\left.\quad a^{-1} w_{1}\left(z, z^{a}, \ldots, z^{a^{4}}\right), b^{-1} w_{2}\left(z, z^{a}, \ldots, z^{a^{4}}\right)\right\rangle .
\end{array}
$$

\section{Acknowledgments}

We would like to thank Collin Bleak for helpful discussions related to [Bleak et al. 2013] and Martin Kassabov for introducing to us the Burnside procedure used in [Guralnick et al. 2011].

\section{References}

[Bestvina and Brady 1997] M. Bestvina and N. Brady, "Morse theory and finiteness properties of groups", Invent. Math. 129:3 (1997), 445-470. MR Zbl

[Bleak and Quick 2017] C. Bleak and M. Quick, "The infinite simple group $V$ of Richard J. Thompson: presentations by permutations”, Groups Geom. Dyn. 11:4 (2017), 1401-1436. MR Zbl

[Bleak et al. 2013] C. Bleak, H. Bowman, A. Gordon Lynch, G. Graham, J. Hughes, F. Matucci, and E. Sapir, "Centralizers in the R. Thompson group $V_{n}$ ", Groups Geom. Dyn. 7:4 (2013), 821-865. MR Zbl

[Brin 2004] M. G. Brin, "Higher dimensional Thompson groups", Geom. Dedicata 108 (2004), 163-192. MR Zbl

[Brin and Squier 2001] M. G. Brin and C. C. Squier, "Presentations, conjugacy, roots, and centralizers in groups of piecewise linear homeomorphisms of the real line", Comm. Algebra 29:10 (2001), 4557-4596. MR Zbl

[Burillo and Cleary 2010] J. Burillo and S. Cleary, "Metric properties of higher-dimensional Thompson's groups", Pacific J. Math. 248:1 (2010), 49-62. MR Zbl

[Cannon et al. 1996] J. W. Cannon, W. J. Floyd, and W. R. Parry, "Introductory notes on Richard Thompson's groups”, Enseign. Math. (2) 42:3-4 (1996), 215-256. MR Zbl

[Fluch et al. 2013] M. G. Fluch, M. Marschler, S. Witzel, and M. C. B. Zaremsky, "The BrinThompson groups $s V$ are of type $\mathrm{F}_{\infty}$ ”, Pacific J. Math. 266:2 (2013), 283-295. MR Zbl

[Geoghegan 2008] R. Geoghegan, Topological methods in group theory, Graduate Texts in Math. 243, Springer, 2008. MR Zbl

[Guralnick et al. 2011] R. M. Guralnick, W. M. Kantor, M. Kassabov, and A. Lubotzky, "Presentations of finite simple groups: a computational approach", J. Eur. Math. Soc. 13:2 (2011), 391-458. MR $\mathrm{Zbl}$

[Hatcher 2002] A. Hatcher, Algebraic topology, Cambridge Univ. Press, 2002. MR Zbl

[Hennig and Matucci 2012] J. Hennig and F. Matucci, "Presentations for the higher-dimensional Thompson groups $n V$ ”, Pacific J. Math. 257:1 (2012), 53-74. MR Zbl

[Higman 1974] G. Higman, Finitely presented infinite simple groups, Notes on Pure Math. 8, Australian National Univ., Canberra, 1974. MR

[Kochloukova et al. 2013] D. H. Kochloukova, C. Martínez-Pérez, and B. E. A. Nucinkis, "Cohomological finiteness properties of the Brin-Thompson-Higman groups $2 \mathrm{~V}$ and $3 \mathrm{~V}$ ", Proc. Edinb. Math. Soc. (2) 56:3 (2013), 777-804. MR Zbl 
[Martínez-Pérez and Nucinkis 2013] C. Martínez-Pérez and B. E. A. Nucinkis, "Bredon cohomological finiteness conditions for generalisations of Thompson groups", Groups Geom. Dyn. 7:4 (2013), 931-959. MR Zbl

[Martínez-Pérez et al. 2016] C. Martínez-Pérez, F. Matucci, and B. E. A. Nucinkis, "Cohomological finiteness conditions and centralisers in generalisations of Thompson's group V", Forum Math. 28:5 (2016), 909-921. MR Zbl

[Quillen 1973] D. Quillen, "Higher algebraic K-theory, I", pp. 85-147 in Algebraic K-theory, I: Higher K-theories (Seattle, 1972), edited by H. Bass, Lecture Notes in Math. 341, Springer, 1973. MR Zbl

[Stein 1992] M. Stein, "Groups of piecewise linear homeomorphisms", Trans. Amer. Math. Soc. 332:2 (1992), 477-514. MR Zbl

Received January 17, 2017. Revised December 12, 2017.

CONCHITA MARTÍNEZ-PÉREZ

DEPARTAMENTO dE MATEMÁticas

UNIVERSIDAD DE ZARAGOZA

ZARAGOZA

SPAIN

conmar@unizar.es

FRANCESCO MATUCCI

Instituto de Matemática, Estatística e ComputaÇão Científica

UNIVERSIDADE ESTADUAL DE CAMPINAS

CAMPINAS

BRAZIL

francesco@ime.unicamp.br

BRITA NUCINKIS

DEPARTMENT OF MATHEMATICS

ROYAL HOLLOWAY

UNIVERSITY OF LONDON

UNITED KINGDOM

brita.nucinkis@rhul.ac.uk 